\title{
Sinusoidal ephrin receptor EPHB4 controls hematopoietic progenitor cell mobilization from bone marrow
}

\author{
Hyeongil Kwak, ${ }^{1}$ Ombretta Salvucci, ${ }^{1}$ Roberto Weigert, ${ }^{2}$ Jorge L. Martinez-Torrecuadrada, ${ }^{3}$ Mark Henkemeyer, ${ }^{4}$ Michael C. Poulos, ${ }^{5}$ \\ Jason M. Butler, ${ }^{5}$ and Giovanna Tosato ${ }^{1}$ \\ 'Laboratory of Cellular Oncology, Center for Cancer Research (CCR), National Cancer Institute (NCI), NIH, and ${ }^{2}$ National Institute of Dental and Craniofacial Research (NIDCR) and Laboratory of Cellular and \\ Molecular Biology, NCI, NIH, Bethesda, Maryland, USA. 3Proteomics Unit, Biotechnology Programme, Centro National de Investigaciones Oncologicas (CNIO), Madrid, Spain. ${ }^{2}$ Department of Developmental Biology, \\ University of Texas Southwestern Medical Center, Dallas, Texas, USA. ํㅗㄹepartment of Cenetic Medicine, Weill Cornell Medical College, New York, New York, USA.
}

\begin{abstract}
Hematopoietic stem and progenitor cells (HSPCs) reside in the bone marrow. Stress signals from cancer and other conditions promote HSPC mobilization into circulation and subsequent homing to tissue microenvironments. HSPC infiltration into tissue microenvironments can influence disease progression; notably, in cancer, HSPCs encourage tumor growth. Here we have uncovered a mutually exclusive distribution of EPHB4 receptors in bone marrow sinusoids and ephrin B2 ligands in hematopoietic cells. We determined that signaling interactions between EPHB4 and ephrin B2 control HSPC mobilization from the bone marrow. In mice, blockade of the EPHB4/ephrin B2 signaling pathway reduced mobilization of HSPCs and other myeloid cells to the circulation. EPHB4/ephrin B2 blockade also reduced HSPC infiltration into tumors as well as tumor progression in murine models of melanoma and mammary cancer. These results identify EPHB4/ephrin B2 signaling as critical to HSPC mobilization from bone marrow and provide a potential strategy for reducing cancer progression by targeting the bone marrow.
\end{abstract}

\section{Introduction}

Bone marrow-derived hematopoietic stem and progenitor cells (HSPCs) and myeloid cells contribute to primary and metastatic tumor growth as they reach the circulation and infiltrate tissues, where they generate "fertile" microenvironments for tumors to grow through various mechanisms (1-5). No effective means currently exist to block the exit of HSPCs from the bone marrow cavity, preventing HSPC entry into circulation $(6,7)$. Development of therapies for blocking HSPC exit from the bone marrow would provide an opportunity for new anticancer strategies.

Most HSPCs reside in the bone marrow in the "stem cell niche," which regulates stem cell functions (7). However, a small proportion of HSPCs physiologically traffic to the blood stream $(8,9)$, and this trafficking is accelerated by many stress signals, including tissue ischemia and cancer $(10,11)$. In cancer, increases in circulating HSPCs are accompanied by HSPC infiltration of tumor tissues and pre-metastatic sites, where they differentiate into pro-tumorigenic myeloid cells $(4,11-13)$. Thus, the bone marrow has emerged as an attractive target for therapies designed to abrogate pathogenic signals arising from bone marrow-derived cells (14).

Effective drugs and protocols have been developed to induce mobilization of HSPCs to the blood as a source of grafts for clinical transplantation $(6,15)$. By contrast, there are no effective drugs or protocols to reduce HSPC mobilization, despite evidence support-

Conflict of interest: J.L. Martinez-Torrecuadrada is the inventor on a patent describing ephrin B2 antibodies.

Submitted: April 1, 2016; Accepted: September 29, 2016.

Reference information: J Clin Invest. 2016;126(12):4554-4568. doi:10.1172/JCI87848. ing the potential benefit of this intervention in cancer and other clinical settings. This is in part due to the complexities of mobilizing signals and to an incomplete understanding of the mechanisms that control HSPC mobilization $(6,7)$. Granulocyte colony stimulating factor (G-CSF), most commonly used for mobilization of HSPCs, does not act directly on HSPCs, but rather affects myeloid cells, which express the specific G-CSF receptor (16). According to current thinking, HSPCs are passive bystanders during bone marrow mobilization by G-CSF, swept out of the bone marrow by a process orchestrated by myeloid cells, which disrupt adhesive bonds holding HSPCs in the bone marrow niche $(6,16-19)$.

We report on the discovery that ephrin B2/EPHB4 signaling critically regulates HSPC exit from the bone marrow and provide evidence that blocking this signaling reduces HSPC mobilization to the blood and suppresses tumor growth.

\section{Results}

EPHB 4 receptor and ephrin $B 2$ ligand are distinctly distributed in bone marrow cells. HSPCs are distributed throughout the bone marrow and preferentially localize adjacent to the sinusoidal blood vessels, a network of fenestrated venules that allows cell trafficking in and out of circulation (20-22). This sinusoidal network, constituting $\sim 30 \%$ of bone marrow and distributed throughout the femoral cavity, forms numerous anastomoses and eventually coalesces into a larger central sinus (Figure 1A) (20-22). We discovered that EPHB4, a transmembrane receptor for the ephrin B2 ligand (23) is present in the bone marrow sinusoidal vessels (Figure 1B). Instead, the Sca- $1^{+}$arterioles, which bring oxygen and nutrients to the bone marrow (20-22), are EPHB $4^{\text {lo/- }}$ (Figure 1B). Costaining for endomucin, a mark- 
A

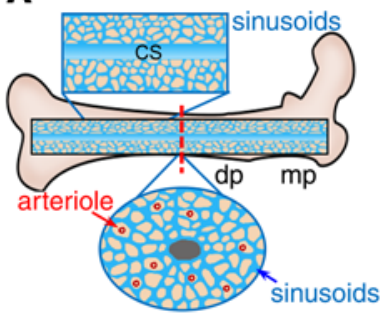

B

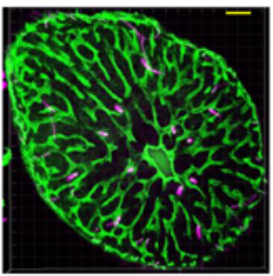

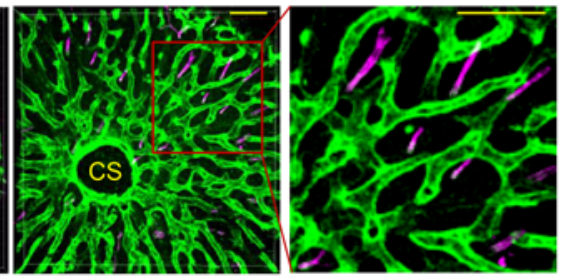

Sca-1

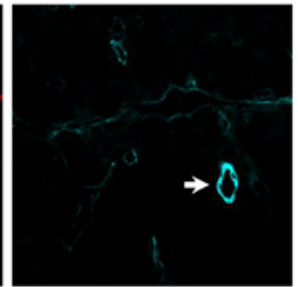

EPHB4

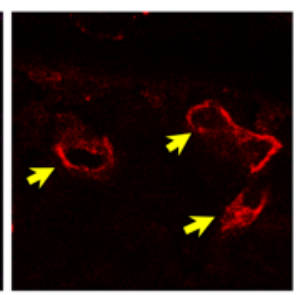

\section{DAPI/OPN/EPHB4}

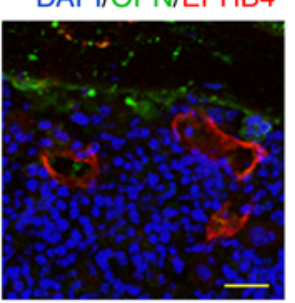

DAPI/Endo/EPHB4/Sca-1

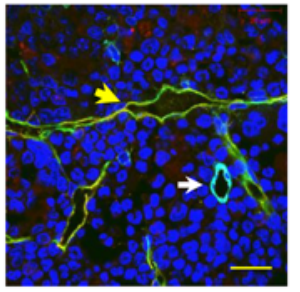

$\pi$
E

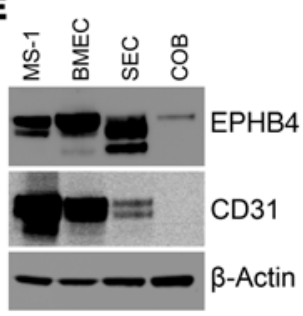

F

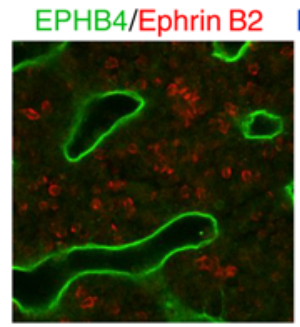

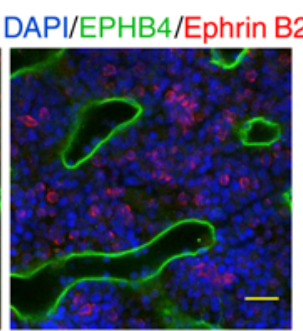

Endomucin

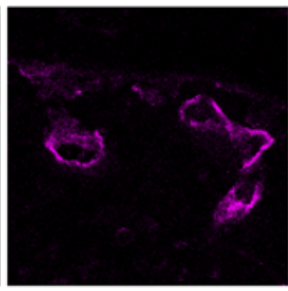

.

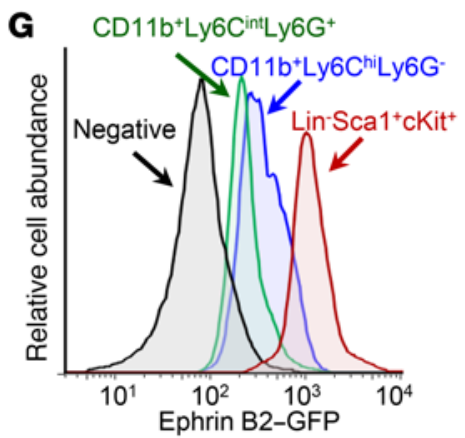

H

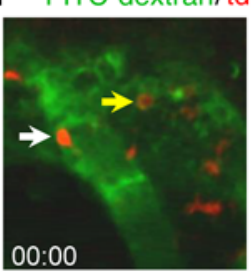

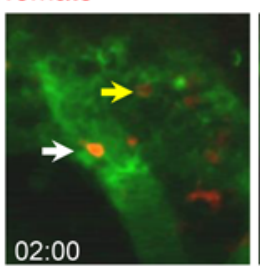

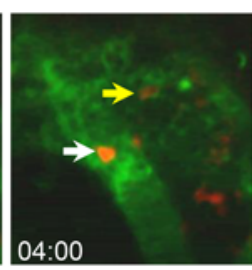

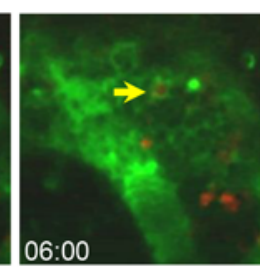
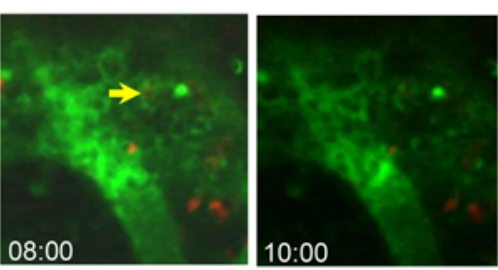

Figure 1. Bone marrow sinusoidal vessels are EPHB4+ephrin B2-, and hematopoietic cells are ephrin B2+EPHB4- (A) Cartoon representation: femur with sinusoidal vessels, diaphysis (dp), metaphysis (mp), and central sinus (CS). Red dotted line indicates position of sections in B-D. (B) Immunofluorescence staining: EPHB4+Sca-1- (green) sinusoidal vessels, Sca-1+EPHB4- (magenta) arterioles, and central sinus; right panel shows a magnification of the middle panel. Scale bars: $100 \mu \mathrm{m}$. (C) Endomucin ${ }^{+}$sinusoidal vessels are EPHB4+Sca-10/- (yellow arrows); Sca- $1^{+}$arterial vessels are endomucin ${ }^{-} E P H B 4^{10 /-}$ (white arrows). Endomucin (Endo, green), EPHB4 (red), and Sca-1 (light blue); DAPI (blue) identifies cell nuclei. Scale bar: $20 \mu \mathrm{m}$. (D) OPN ${ }^{+}$osteoblasts are endomucin ${ }^{10 /-E P H B} 4^{10 /-}$ (white arrows); endomucin ${ }^{+}$sinusoidal vessels are EPHB4+OPN- (yellow arrows). OPN (green); endomucin (magenta); EPHB4 (red); DAPI (blue). Scale bar: $50 \mu \mathrm{m}$. (E) EPHB4 and CD31 in lysates of the endothelial (MS-1 and BMEC) mouse cell lines, primary sinusoidal endothelial cells (SEC), and primary calvarial osteoblasts (COB); membrane reprobed for $\beta$-actin. (F) Distribution of ephrin B2+EPHB4- cells (red) surrounding EPHB4 ${ }^{+}$ephrin

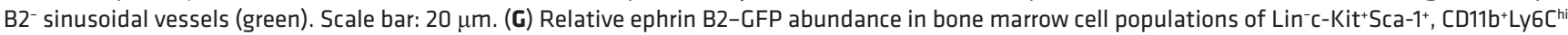
$\mathrm{Ly}_{6 \mathrm{C}}{ }^{-}$, and $\mathrm{CD} 11 \mathrm{~b}^{+} \mathrm{Ly} 6 \mathrm{C}^{\mathrm{int}} \mathrm{Ly} 6 \mathrm{C}^{+}$from an EfnB2 ${ }^{\mathrm{H} 2 \mathrm{BCFP}}$ reporter mouse by flow cytometry. (H) Intravital time-lapse imaging of calvarium showing the timedependent attachment of tdTomato-fluorescent (red) cells to the outside wall of sinusoidal vessels, followed by their disappearance. White and yellow arrows point to two fluorescent cells initially on or lateral to sinusoidal vessels that later disappear from view. Sinusoidal vessels: green (FITC-dextran was used to perfuse vessels). 
er for bone marrow sinusoids (24), confirmed this pattern: the endomucin ${ }^{+}$bone marrow sinusoids were EPHB4 $4^{+}$; the endomu$\mathrm{cin}^{-/ \mathrm{lo}} \mathrm{Sca}-1^{+}$arterioles were EPHB $4^{\mathrm{lo} /-}$ (Figure 1C and Supplemental Figure 1, A and B; supplemental material available online with this article; doi:10.1172/JCI87848DS1). The DAPI ${ }^{+}$cells surrounding the sinusoids, likely hematopoietic cells, were also EPHB4- (Figure 1C). Osteoblasts have previously been reported to express $\operatorname{EPHB} 4(25,26)$. By immunohistochemistry, we found that osteopontin ${ }^{+}\left(\mathrm{OPN}^{+}\right)$osteoblasts were EPHB4 $4^{\text {lo }}$ (Figure 1D). However, compared with primary bone marrow sinusoidal endothelial cells, primary osteoblasts $\left(\mathrm{OPN}^{+} \mathrm{Sp}^{+}\right.$, Supplemental Figure 1, C and D; expressing $S p 7$ and osteocalcin mRNAs, Supplemental Figure 1E) expressed substantially less Ephb4 mRNA (Supplemental Figure 1E) and protein (Figure 1E).

Since EPHB4 receptors uniquely bind to ephrin B2 ligands (23), we examined whether bone marrow hematopoietic cells are ephrin $\mathrm{B} 2^{+}$. We detected widespread ephrin B2 expression in cells surrounding the ephrin $\mathrm{B}^{-}$sinusoids by specific immunostaining (Figure $1 \mathrm{~F}$ ). Taking advantage of the $E f n b 2^{\text {H2BGFP }}$ reporter mouse, in which nuclear GFP signal reflects ephrin B2 expression because the $H 2 B G F P$ gene is knocked into the ephrin B2 locus (27), flow cytometry showed that the $\mathrm{Lin}^{-} \mathrm{c}-\mathrm{Kit}^{+} \mathrm{Sca}-1^{+} \mathrm{HSPC}$ expressed more ephrin $\mathrm{B} 2$ than the $\mathrm{CD} 11 \mathrm{~b}^{+} \mathrm{Ly} 6 \mathrm{C}^{\text {hi }} \mathrm{Ly}_{6 \mathrm{G}^{-}}$monocytes and the CD11b ${ }^{+} \mathrm{Ly}-$ $6 \mathrm{C}^{\text {int }} \mathrm{Ly} 6 \mathrm{G}^{+}$neutrophils, as suggested by the relative mean GFP fluorescence intensities in these populations (Figure $1 G$ ).

EPHB4 only binds to ephrin B2, but ephrin B2 can also bind the EPHB1, EPHB2, and EPHA4 receptors (23). We found that the endomucin ${ }^{+} \mathrm{EPHB} 4^{+}$bone marrow sinusoids were EPHB1EPHB2-, and EPHA4- (Supplemental Figure 1, B and F). Thus, bone marrow HSPCs, which predominantly reside proximal to the sinusoidal vessels $(22,28)$, are ephrin $\mathrm{B}^{+} \mathrm{EPHB} 4^{-}$and sinusoidal vessels are EPHB4 ${ }^{+}$ephrin B2-EPHB1-EPHB2-EPHA4- Since EPHB4 and ephrin B2 are transmembrane proteins, these results raised the possibility of signaling interaction between ephrin $\mathrm{B}^{+}$ HSPCs and adjacent EPHB4 ${ }^{+}$sinusoidal endothelium.

In vivo imaging has demonstrated that HSPCs home to the bone marrow through the bone marrow sinusoids (29), but to our knowledge, no such evidence exists for HSPCs exiting the bone marrow. We transplanted fluorescent Lin ${ }^{-}$Sca- $1^{+}$HSPCs sorted from the bone marrow of tandem dimer Tomato (tdTomato) mice (ubiquitously expressing the red fluorescent protein tdTomato) into a WT recipient to populate the recipient bone marrow with fluorescent $\mathrm{Lin}^{-} \mathrm{Sca}-\mathrm{1}^{+}$cells, and subsequently induced hematopoietic cell mobilization from the bone marrow to the peripheral circulation with G-CSF (16). Using intravital time-lapse imaging of the mouse calvarium (29), we documented the movement of tdTomato fluorescent cells from the bone marrow extravascular space to the intravascular sinusoidal space, followed by the disappearance from view of the fluorescent cells attributable to the hemodynamic shear exerted by the flowing blood (Figure $1 \mathrm{H}$ and Supplemental Video 1). These results provide evidence that the sinusoidal vessel wall is a port for hematopoietic cell exit from the bone marrow to the circulation.

EPHB4 controls the transendothelial migration of hematopoietic cells. To test for the possibility that ephrin B2/EPHB4 regulate transendothelial migration of hematopoietic cells, we selected murine endothelial cells that express EPHB4 but not ephrin B2, similar to the bone marrow sinusoidal endothelium. The bone marrow endothelial cells (BMECs) (30) and the pancreatic endothelial SV40-transformed MS-1 cells expressed EPHB4 protein but not ephrin B2 protein (Figure 2A). BMECs and MS-1 cells additionally expressed EPHB1, EPHB2, and EPHA4 to varying degrees, but the bone marrow sinusoids did not (Figure 2A and Supplemental Figure 1B).

Initial experiments revealed that endothelial monolayers substantially favored Transwell migration of bone marrow CD11b ${ }^{+}$Ly6C ${ }^{\text {hi }}$ Ly6G $^{-}$monocytes and Lin $^{-}$c-Kit ${ }^{+}$Sca-1 ${ }^{+}$HSPCs from untreated and G-CSF-treated mice, and to a lesser degree the transmigration of $\mathrm{CD} 11 \mathrm{~b}^{+} \mathrm{Ly} 6 \mathrm{C}^{\text {int }} \mathrm{Ly} 6 \mathrm{G}^{+}$neutrophils (MS-1 monolayer is compared with gelatin only-coated membranes; Supplemental Figure 2, A and B).

We successfully silenced EPHB4 in BMECs (Figure 2B) and MS-1 cells (Supplemental Figure 2C) with lentiviral vector shEPHB4\#1 (sh\#1) or shEPHB4\#2 (sh\#2), without a change in cell morphology, viability, or proliferation (data not shown). We found that EPHB4 silencing significantly reduced the trans-BMEC migration of bone marrow cells (all cells), particularly of monocytes and HSPCs (identified phenotypically as Lin $^{-} \mathrm{c}-\mathrm{Kit}^{+} \mathrm{Sca}-\mathrm{1}^{+}$and functionally as colony-forming precursors), but not the transmigration of neutrophils, T lymphocytes, or B lymphocytes from G-CSF-treated and untreated mice (Figure 2, C-I). These results were largely reproduced in MS-1 cells (Supplemental Figure 2, D-G). Thus, EPHB4 contributes to the transmigration-promoting activity of endothelial monolayers.

EPHB4 and ephrin B2 can each initiate intracellular signaling (EPHB4-derived or ephrin B2-derived) as a consequence of cellto-cell interaction between ephrin $\mathrm{B}^{+}{ }^{+}$and EPHB $4^{+}$cells (23). To distinguish between these mutually exclusive possibilities, we transduced BMECs with WT EPHB4 or a kinase-dead mutant EPHB4 (K647R/kdEPHB4; contains a single K647R mutation in the kinase domain), which acts as a dominant negative for the endogenously expressed EPHB4 while retaining the ability to activate ephrin B2 signaling (31). Compared with WT EPHB4, the mutant EPHB4 K647R significantly impaired transmigration of monocytes and HSPCs, and to a lesser degree the transmigration of neutrophils (Figure 2, C-F), but minimally altered lymphocyte transmigration (Figure 2, $\mathrm{G}$ and $\mathrm{H}$ ), providing evidence for a role of EPHB4 signaling in this in vitro system.

Mechanistically, we found that EPHB4 insignificantly modulates vascular permeability to Texas red-dextran in vitro (Figure 2J) but critically contributes to HSPC adhesion to BMEC monolayers (Figure 2K), as reflected by functional analysis of control and mutant BMEC monolayers where EPHB4 was silenced or a signaling-deficient EPHB4 was expressed. Consistent with EPHB4 regulating hematopoietic cell adhesion to bone marrow sinusoidal endothelium, we detected EPHB4 clustering at points of sinusoidal endothelial cell contact with hematopoietic cells, away from the inner endothelial cell surface lining the lumen of the endomucin $^{+}$sinusoid (Figure 2L). This EPHB4 clustering was not or rarely observed without G-CSF mobilization or in the presence of systemic TNYL-RAW blocking peptides in G-CSF-mobilized mice (data not shown). EPHB4 clustering is induced by ephrin B2 ligand binding through cell-to-cell contact, which promotes adhesion and other signals (32). Since cell adhesion to endothelium is 
A
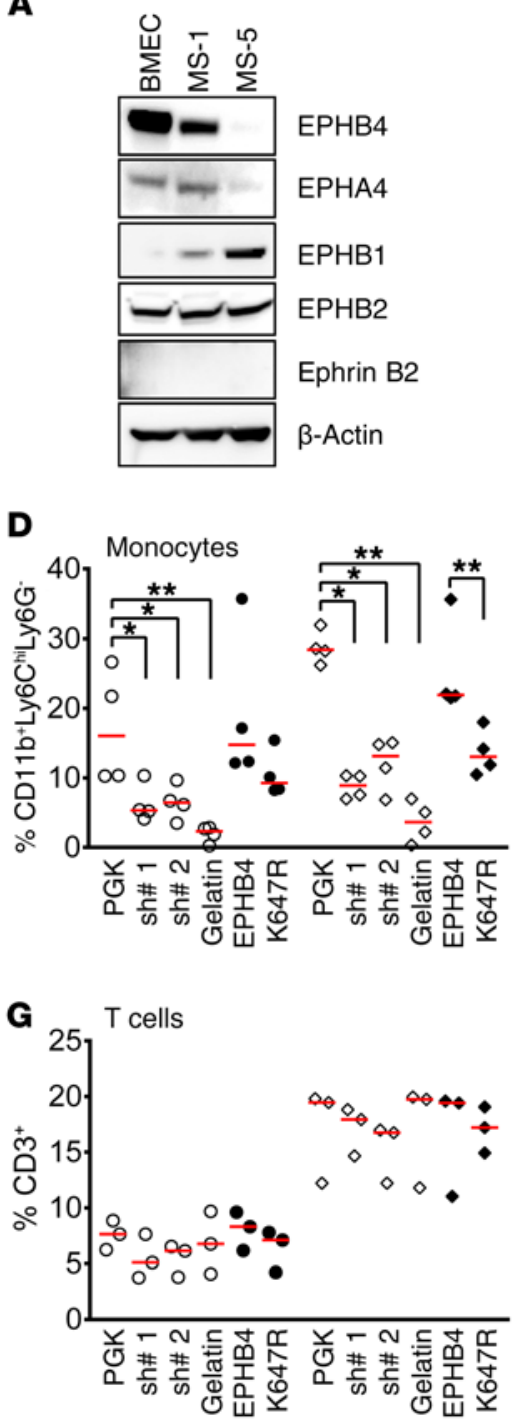

B

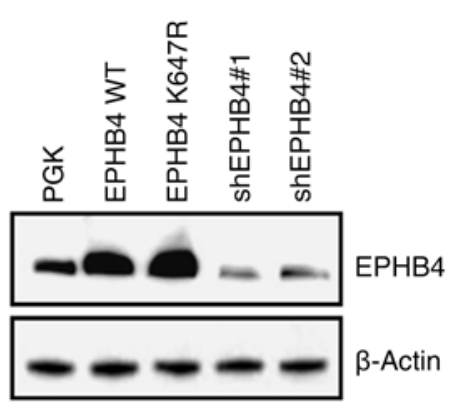

E
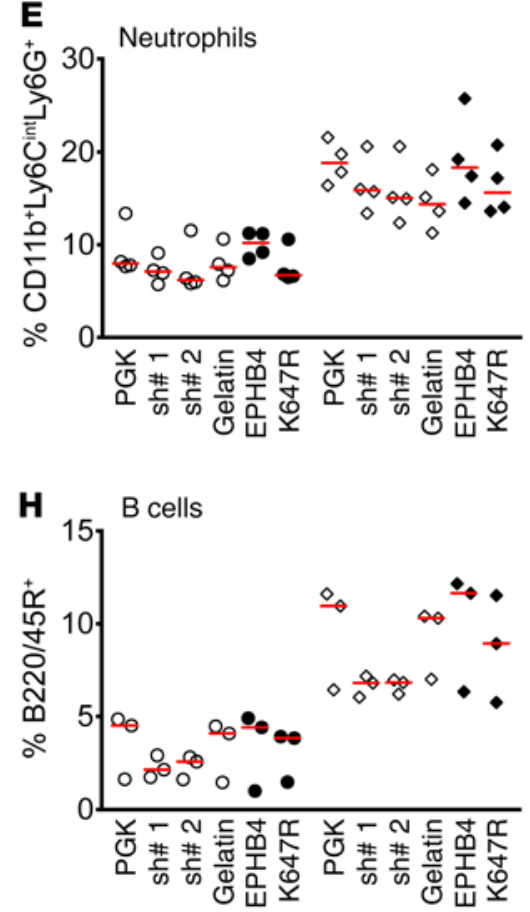

C $\quad 00$ Control

$\diamond \diamond \mathrm{G}-\mathrm{CSF}$
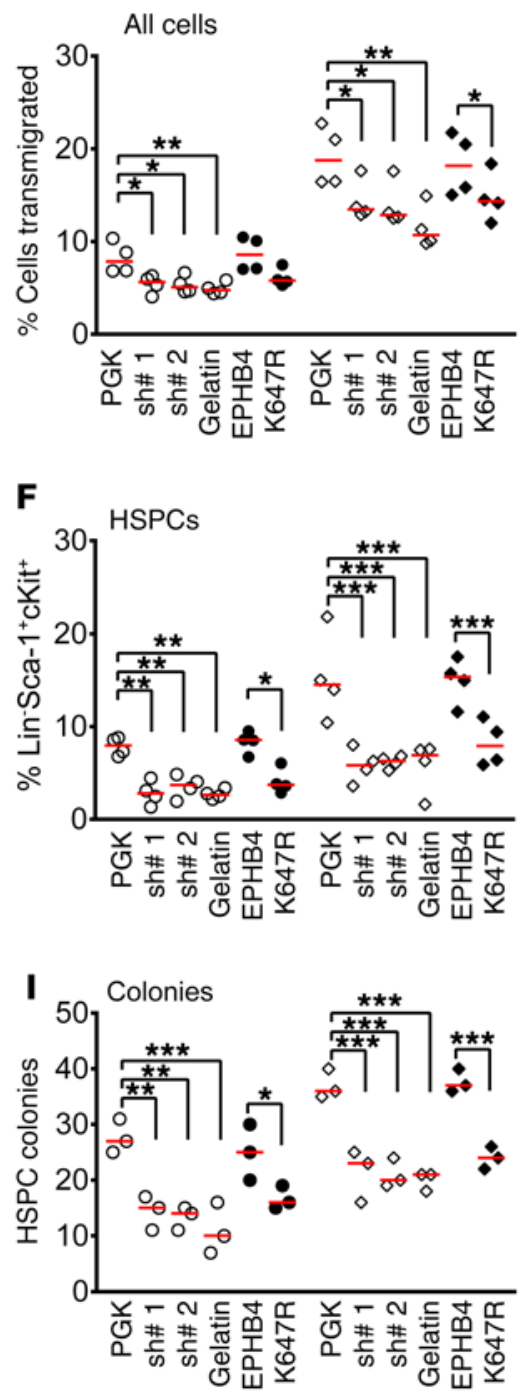
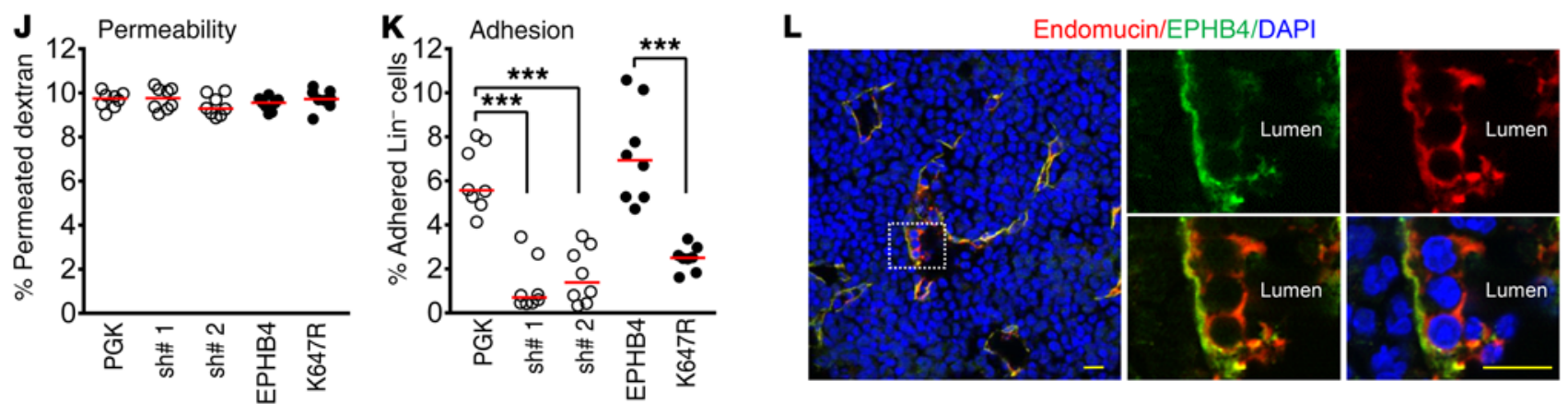

Figure 2. EPHB4 promotes hematopoietic cell transendothelial migration. (A) EPHB1-4 and ephrin B2 in lysates of endothelial (BMEC and MS-1) and stromal (MS-5) mouse cell lines by immunoblotting; membrane reprobed for $\beta$-actin. (B) WT or K647R mutant EPHB4 expression; EPHB4 silencing with sh\#1 and sh\#2 in BMECs; PGK vector was used as a control. Immunoblotting of cell lysates. (C-H) Transendothelial migration of bone marrow hematopoietic cells from G-CSF-treated or control mice through: control (PGK), EPHB4-silenced (sh\#1 and sh\#2), WT EPHB4- (EPHB4), or EPHB4 K647R- (K647R) expressing BMECs; gelatin-coated Transwells were controls. The proportion of monocytes, neutrophils, Lin'c-Kit ${ }^{+} S c a-1^{+}$cells, T cells, and B cells in the cell population loaded into the upper chamber and recovered in the bottom chamber was measured by flow cytometry. Results show \% cells (of total loaded) recovered after transmigration (median of 3-4 independent experiments; red lines). (I) Number of colonies in methylcellulose generated by $100 \mu \mathrm{l}$ suspension of transmigrated cells ( $n=3$ experiments, each performed in triplicate). (J) BMEC monolayer permeability to Texas red-dextran; the results (\% fluorescence/total loaded) reflect means of individual measurements $(n=8)$. (K) Adherence of tdTomato bone marrow Lin- cells to BMEC monolayers; the results (\% cells/total loaded) reflect means of individual measurements $(n=8)$. (L) Distribution of EPHB4 (green) in endomucin ${ }^{+}($red) sinusoidal endothelium showing EPHB4 clustering toward the hematopoietic cells and away from the sinusoidal lumen. Nuclei: DAPI (blue). Boxed area is magnified on the right. Scale bars: $10 \mu \mathrm{m}$. Statistical significance by 1-way ANOVA with Dunnett's multiple comparison test: ${ }^{*} P<0.05 ;{ }^{*} P<0.01 ;{ }^{* * *} P<0.001$. 
A

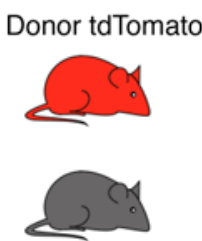

Recipient WT

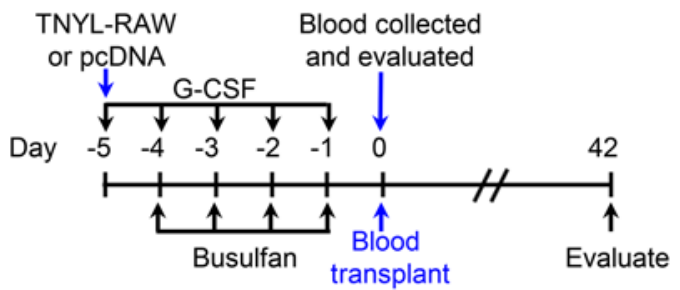

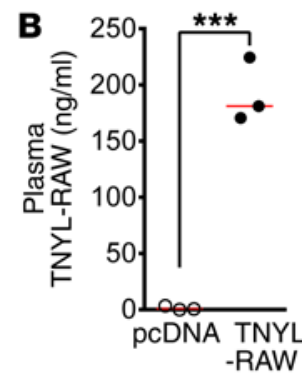

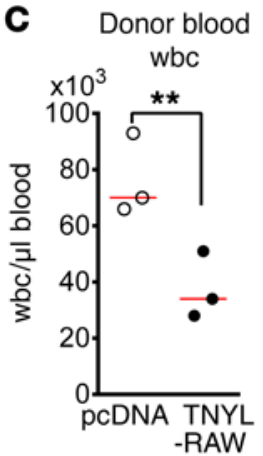

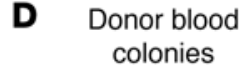

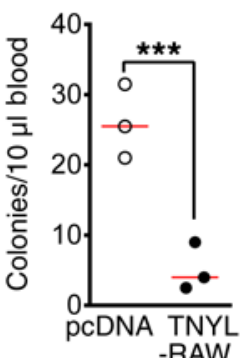

E AMD-treated blood wbc

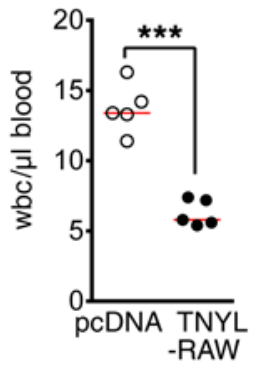

F AMD-treated blood colonies

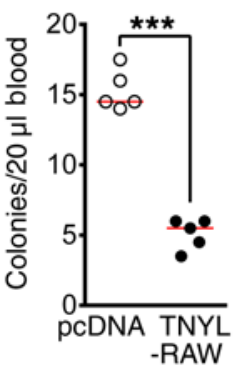

G Constitutive

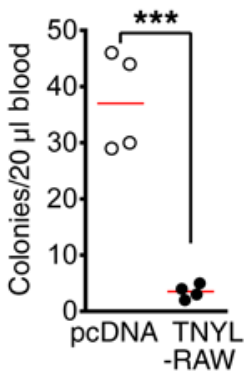

Figure 3. Ephrin B2/EPHB4 interaction is required for hematopoietic cell mobilization. (A) Schematic of experiment. Donor tdTomato mice inoculated with TNYL-RAW or pcDNA expression vectors and treated with G-CSF for 5 days. Donor blood was evaluated and transplanted into WT busulfanpretreated (4 days) recipients. Recipient mice were evaluated on day 42 after transplant. (B-D) G-CSF-mobilized donor mice: plasma concentrations of TNYL-RAW peptide (B); wbc/10 $\mu$ l blood (C); methylcellulose-based colonies/10 $\mu \mathrm{l}$ blood (D); median is indicated by the horizontal lines; $n=3 /$ group. (E and F) AMD3100-mobilized mice: wbc/10 $\mu \mathrm{l}$ blood (E); methylcellulose-based colonies/20 $\mu \mathrm{l}$ blood (F); median is indicated by the horizontal lines; $n=5$ /group. (C) Non-mobilized mice: constitutive methylcellulose-based colonies $/ 400 \mu \mathrm{l}$ blood; median is indicated by the horizontal lines; $n=4 / \mathrm{group}$. $P$ values by Student's $t$ test: ${ }^{* *} P<0.01,{ }^{* *} P<0.001$.

a critical step in transendothelial transmigration (33), these results support a role of activated sinusoidal EPHB4 in the mobilization of hematopoietic cells to the circulation, which uniquely occurs through the bone marrow sinusoidal vessels (28).

EPHB4 signaling controls bone marrow HSPC mobilization to the blood. Since EPHB4 marks the bone marrow sinusoidal vessels and endothelial EPHB4 signaling contributes to the transendothelial migration of ephrin $\mathrm{B} 2^{+}$hematopoietic cells in vitro, we tested whether sinusoidal EPHB4 contributes to the mobilization and/or homing of ephrin $\mathrm{B}^{+}$hematopoietic cells in vivo.

To evaluate a potential role of EPHB4 in HSPC mobilization (Figure 3A, schematic of experiment), we induced robust expression of the EPHB4/ephrin B2 blocking peptide TNYL-RAW (34) in donor mice by hydrodynamic inoculation (35) of a TNYL-RAW expression vector (control mice received the empty pcDNA vector); on the same day, we started treatment with G-CSF (days -5 through -1) to force bone marrow mobilization (16). HSPC mobilization to the blood was measured by (a) counting the number of circulating colony-forming precursors and (b) assessing hematopoietic chimerism in recipient WT mice transplanted with the blood (on day 42 after transplant). The recipient mice were conditioned with a non-ablative regimen of busulfan (days -4 through -1 ) prior to blood transplant (36). To distinguish donor from recipient cells, we utilized tdTomato mice as donors and WT mice as recipients.

The TNYL-RAW-transduced tdTomato donor mice displayed high plasma levels of TNYL-RAW peptides on days 1 through 10 after transduction (Supplemental Figure 3A). On the fifth day (day 0 in the schematic), TNYL-RAW peptide levels were high in TNYL-RAW-transduced mice but were undetected in controls (Figure 3B, experiment 1, and Supplemental Figure 3B confirmatory experiments 2 and 3). Additional characterization of the donor mice at this time point (day 0 in the schematic) showed that the G-CSF-mobilized mice expressing TNYL-RAW displayed significantly lower wbc counts compared with the controls (Figure 3C and Supplemental Figure 3C), but the distribution of lymphocytes, neutrophils, and monocytes was similar (Supplemental Figure 3D). Strikingly, the TNYL-RAW-expressing mice had significantly fewer circulating HSPCs than controls, as reflected by colony counts (Figure 3D and Supplemental Figure 3E). We could not accurately quantitate the $\mathrm{Lin}^{-} \mathrm{c}-\mathrm{Kit}^{+} \mathrm{Sca}-{ }^{+}$cells in blood due to the paucity of $\mathrm{Lin}^{-} \mathrm{c}-\mathrm{Kit}^{+}$cells in blood (Supplemental Figure 3, F and G), attributed to G-CSF-induced c-Kit cleavage from the cell surface of HSPCs (37).

To investigate whether these effects of TNYL-RAW peptides were specific to G-CSF-induced mobilization, we examined hematopoietic cell mobilization induced by AMD3100, which specifically blocks the cytokine receptor CXCR4 (schematic of experiment, Supplemental Figure 3H) (38). We also examined constitutive HSPC mobilization. AMD3100-mobilized mice expressing systemic TNYL-RAW peptides $(178 \pm 5.2 \mathrm{ng} / \mathrm{ml}$ plasma) displayed lower wbc counts (Figure $3 \mathrm{E}$ ), with no change in the distribution of cell types (data not shown) and lower numbers of circulating colony-forming HSPC precursors (Figure 3F) compared with controls. In addition, non-mobilized mice that 


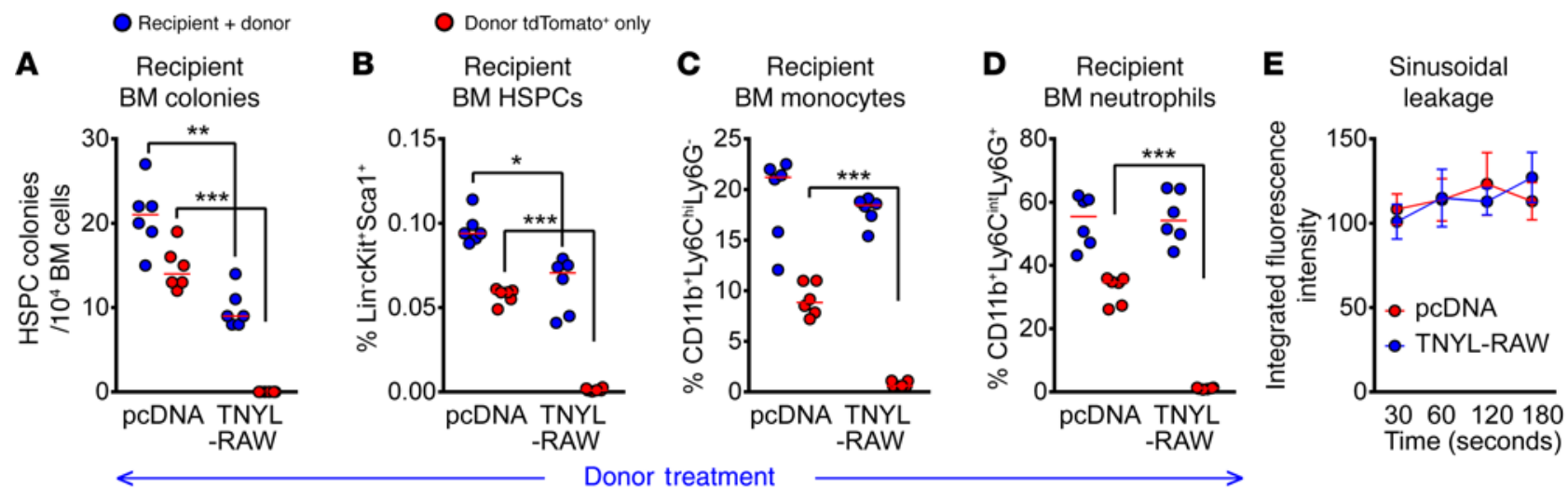

$\mathbf{F}$
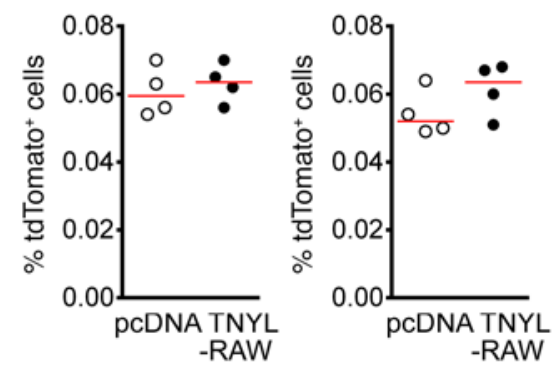

Recipient treatment

Donor treatment
G

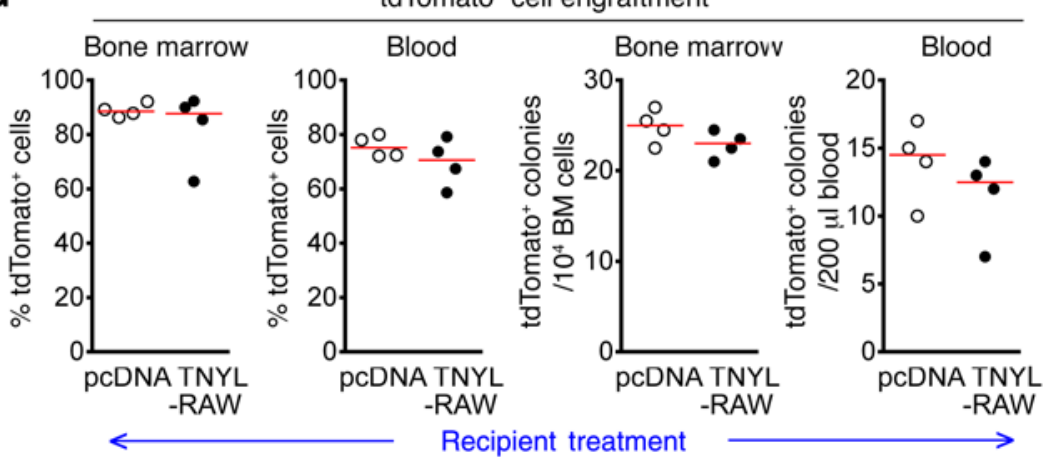

Figure 4. Ephrin B2/EPHB4 blockade reduces hematopoietic cell mobilization but not homing to the bone marrow. (A-D) Bone marrow from WT recipient mice transplanted with blood from G-CSF-mobilized donor tdTomato mice (blood from each donor mouse transplanted into 2 recipient mice; $n=6$ recipients/group). Blue dots: all cells (donor + recipient); red dots: donor only-derived cells. (A) Methylcellulose colonies from $10^{4} \mathrm{BM}$ cells; median (red lines); (B) HSPCs; median \% (red lines)/all bone marrow cells. (C) Monocytes; median \% (red lines)/all BM cells. (D) Neutrophils; median \% (red lines)/ all BM cells. $P$ values by Student's $t$ test: ${ }^{*} P<0.05$, ${ }^{* *} P<0.01$, ${ }^{* * *} P<0.001$. (E) Sinusoidal leakage of Texas red-dextran in TNYL-RAW- and pcDNAexpressing mice $(n=3)$. Intravital imaging measurements shown as time traces of fluorescent signal; the results reflect the mean \pm SD (error bars). (F) tdTomato ${ }^{+}$BM Lin ${ }^{-}$HSPC homing: (left) cells from untreated donors injected into recipients expressing TNYL-RAW or pcDNA; (right) cells from donors expressing TNYL-RAW or pcDNA injected into WT untreated recipients; median (red lines; $n=4$ /group). (C) tdTomato ${ }^{+}$BM engraftment (day 42 after transplant) into recipients expressing TNYL-RAW or pcDNA; \% donor-derived cells in bone marrow and blood and number of donor-derived methylcellulose colonies in recipient $\mathrm{BM}$ and blood. The results reflect the median (red lines; $n=4 /$ group).

expressed systemic TNYL-RAW peptides $(225 \pm 11.9 \mathrm{ng} / \mathrm{ml})$ displayed a lower number of circulating colony-forming HSPC precursors compared with controls (Figure 3G), providing evidence that EPHB4/ephrin B2 blockade reduces HSPC exit from the bone marrow to the circulation by diverse stimuli.

This reduction in HSPCs in blood of TNYL-RAW mice was mirrored by the results of transplant experiments. Specifically, we found that recipients of blood from TNYL-RAW-expressing mice mobilized with G-CSF (evaluated on day 42 after transplant, Figure $3 \mathrm{~A}$ ) displayed significantly fewer colony-forming precursors in the bone marrow compared with the recipients of blood from control (pcDNA-transduced) mice; remarkably, these colonies were almost entirely of recipient origin, as they lacked the tdTomato fluorescence expected from donor-derived cells (Figure $4 \mathrm{~A}$ and Supplemental Figure 4A). Consistent with this, the td Tomato ${ }^{+}$Lin $^{-} \mathrm{c}-\mathrm{Kit}^{+} \mathrm{Sca}-\mathrm{1}^{+}$HSPCs (Figure 4B and Supplemental Figure 4B), monocytes (Figure 4C and Supplemental Figure 4C), and neutrophils (Figure 4D and Supplemental Figure 4D) were virtually absent from the bone marrow of mice transplanted with blood from TNYL-RAW-expressing mice, but numerous in the bone marrow of mice transplanted with blood from control mice.
Analysis of recipients' blood after G-CSF mobilization on days 42-46 after transplantation confirmed the absence of tdTomato ${ }^{+}$ HSPCs in blood (Supplemental Figure 4E).

The bone marrows of G-CSF-mobilized TNYL-RAW-expressing mice was indistinguishable from that of control-transduced mice with respect to total cell number/femur, number of HSPC

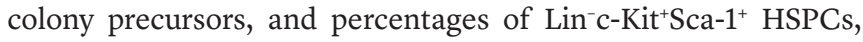
monocytes, and neutrophils (Supplemental Figure 4, F-J), consistent with the bone marrow broad cell reserve and capacity to adapt to mobilizing signals. In addition, no differences were noted in sinusoidal blood vessel permeability in control and TNYL-RAWexpressing mice $(239 \pm 161 \mathrm{ng} / \mathrm{ml})$, as assessed by intravital calvarial bone imaging after Texas red-dextran injection (Figure 4E).

To evaluate a potential role of EPHB4 in HSPC homing to the bone marrow, we tested the effects of EPHB4/ephrin B2 blockade in recipients and donors of HSPCs (schematic of experiments, Supplemental Figure 4, K and L). Homing to the bone marrow was measured by flow cytometry 15 hours after tail vein inoculation of HSPC-enriched bone marrow cells (39). We found that the homing of tdTomato ${ }^{+}$HSPC-enriched bone marrow cell populations was similar in control and TNYL-RAW-expressing (182 \pm 7.4 
A

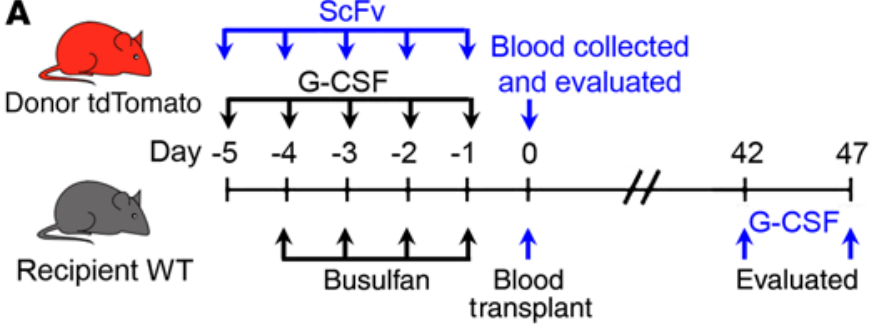

B Donor blood wbc

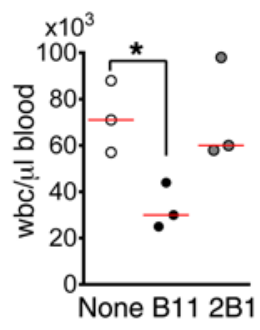

C Donor blood differentials

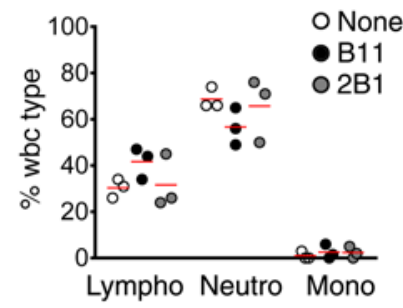

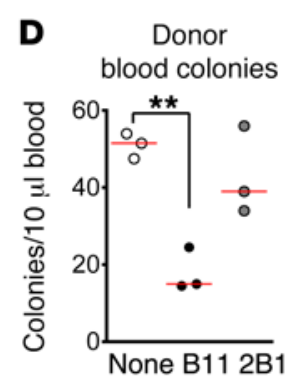

Recipient + donor

\section{E}

Recipient BM HSPCs

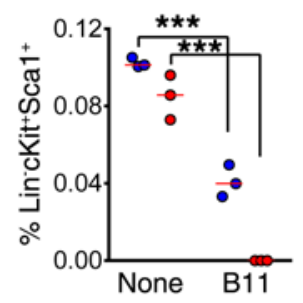

O Donor tdTomato only

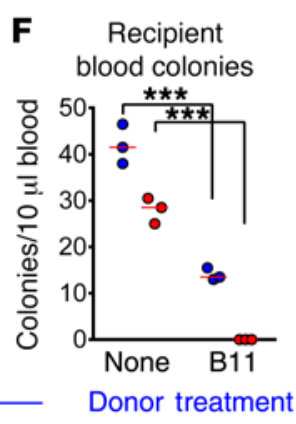

\section{G}

Recipient BM monocytes

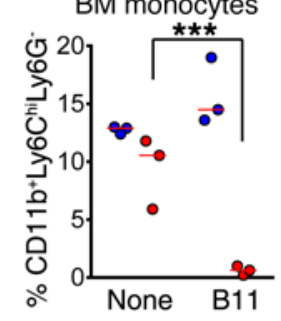

H Recipient BM neutrophils

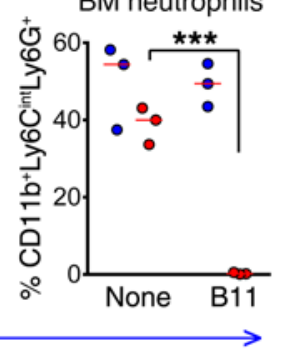

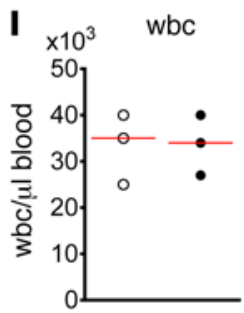
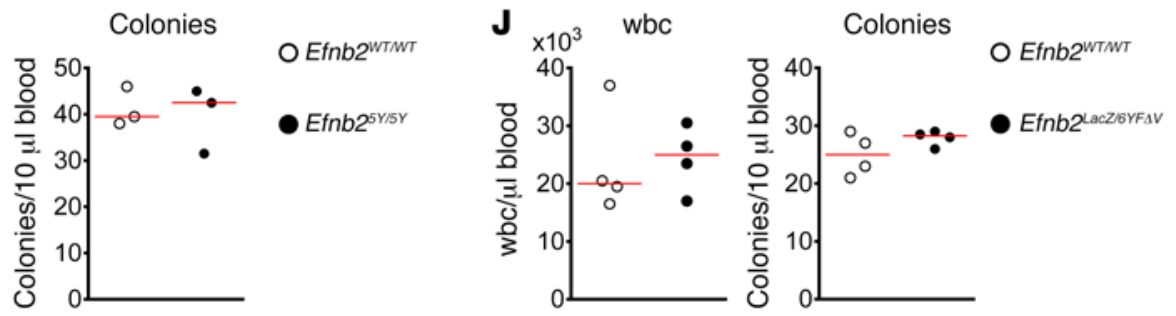

Figure 5. Ephrin B2 neutralization reduces hematopoietic cell mobilization attributable to reduced EPHB4 signaling. (A) Schematic of experiment. Donor tdTomato mice were treated with G-CSF alone or with scFv antibodies (day -5 to -1 ). Day 0 : blood from donor mice was evaluated and transplanted into busulfan-pretreated WT recipients evaluated on days 42 or 47 (G-CSF was injected on days 42-46). (B-D) Peripheral blood from G-CSF-mobilized donor tdTomato mice (B11 or 2B1 scFv antibody-treated); control mice: no antibodies ( $n=3 /$ group). (B) wbc; median (red lines). (C) Differential counts; \% median indicated by red lines. Lympho, lymphocytes; Neutro, neutrophils; Mono, monocytes. (D) Colonies; median (red lines). Statistical significance by 1-way ANOVA with Dunnett's multiple comparison test: ${ }^{*} P<0.05$, ${ }^{* *} P<0.01$. (E-H) Evaluation of WT recipient mice transplanted with blood from donor tdTomato mice ( $n=3$ recipients/ group). Blue dots: all (donor + recipient) cells; red dots: donor only-derived cells. (E) BM HSPCs; day 42 after transplant; $n=3 /$ group; median \% (red lines)/all bone marrow cells. (F) Colonies from blood; day 47 after transplant (G-CSF treatment days 42 through 46); $n=3 /$ group; median (red lines). (C) BM monocytes; day 42 after transplant; $n=3$ /group; median \% (red lines)/all BM cells. (H) BM neutrophils; day 42 after transplant; $n=3 /$ group; median \% (red lines)/all BM. $P$ values by Student's $t$ test: ${ }^{* *} P<0.001$. (I and J) G-CSF mobilization in mice with ephrin B2 signaling deficiencies. Blood wbc and colony-forming precursors in $E f n b 2^{5 / 5 V}$ and $E f n b 2^{W T / W T}$ littermates; $n=3 /$ group (I); and in Efnb2 $2^{\text {LacZ/6YFAV }}$ and $E f n b 2^{W T / W T}$ littermates; $n=4 /$ group (median: red lines) (J).

$\mathrm{ng} / \mathrm{ml}$ plasma) recipient mice (Figure $4 \mathrm{~F}$, left). Also, the homing of td Tomato ${ }^{+}$HSPC-enriched bone marrow cell populations from TNYL-RAW-expressing ( $178 \pm 5.4 \mathrm{ng} / \mathrm{ml}$ plasma) and control donor mice occurred normally in untreated recipients (Figure $4 \mathrm{~F}$, right). In addition, engraftment of HSPC-enriched bone marrow cells (measured on day 42 after transplant) occurred normally in TNYLRAW-expressing recipients as reflected by recovery of tdTomato ${ }^{+}$ cells and colony-forming HSPC precursors in bone marrow and blood (Figure 4G). Circulating levels of TNYL-RAW blocking peptides were expectedly high at the time of transplant $(255 \pm 40.5 \mathrm{ng} /$ $\mathrm{ml}$ plasma); on day 42 after transplant, circulating levels of TNYLRAW were lower $(40.5 \pm 4.1 \mathrm{ng} / \mathrm{ml}$ plasma). These results provide evidence that EPHB4/ephrin B2 critically regulate bone marrow HSPC mobilization but not the homing and engraftment of HSPCs to the bone marrow. These results are consistent with homing and mobilization being mechanistically distinct processes (40).
In additional experiments, we employed two single-chain fragment variable ( $\mathrm{scFv}$ ) antibodies to ephrin B2: B11, which prevents ephrin B2 binding to EPHB4, and 2B1, which does not (41). A schematic of the experiment is shown in Figure 5A. The antibody regimen (daily injections; $4 \mathrm{mg} / \mathrm{mouse}$ on days -5 through -1 ) was based on biodistribution studies in mice with fluorescent-labeled B11 and 2B1 antibodies (41). Similar to the results with TNYL-RAW blocking peptides, administration of the scFv ephrin B2-neutralizing B11 antibody significantly reduced blood wbc compared with the controls, which received either no antibody treatment or treatment with scFv 2B1 antibody (Figure 5B and Supplemental Figure $5 \mathrm{~A})$, without a significant change $(P>0.05)$ in the distribution of lymphocytes, monocytes, and neutrophils (Figure 5C and Supplemental Figure 5B). Also, B11 antibody treatment significantly reduced the number of blood HSPCs as measured by colony formation (Figure 5D and Supplemental Figure 5C). 
A

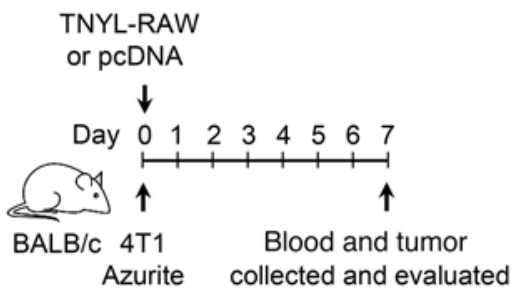

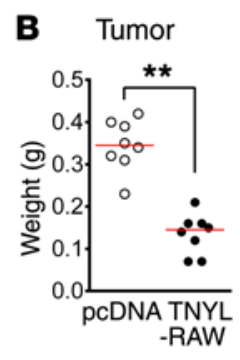
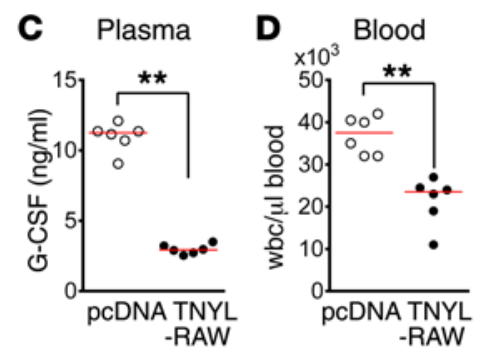

E

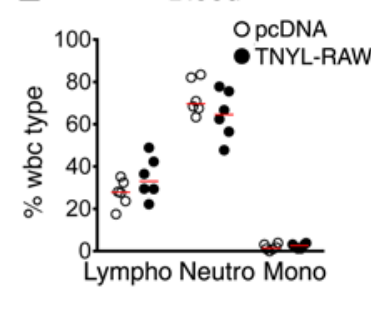

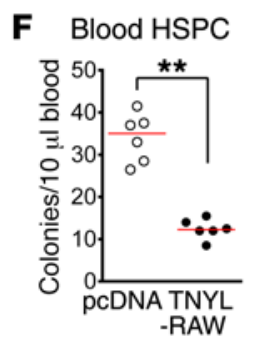
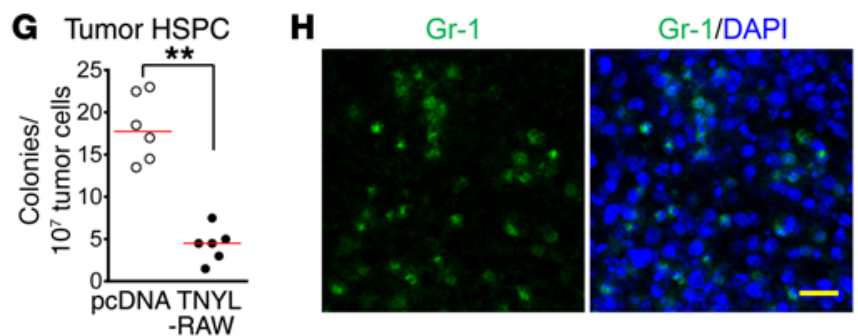

I Tumor $\mathrm{Gr}-\mathbf{1}^{+}$cells

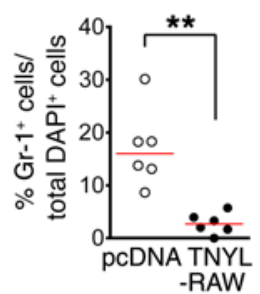

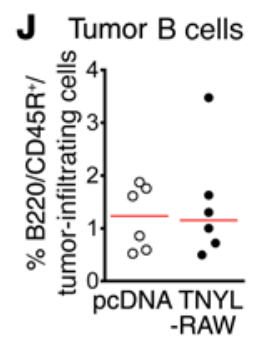
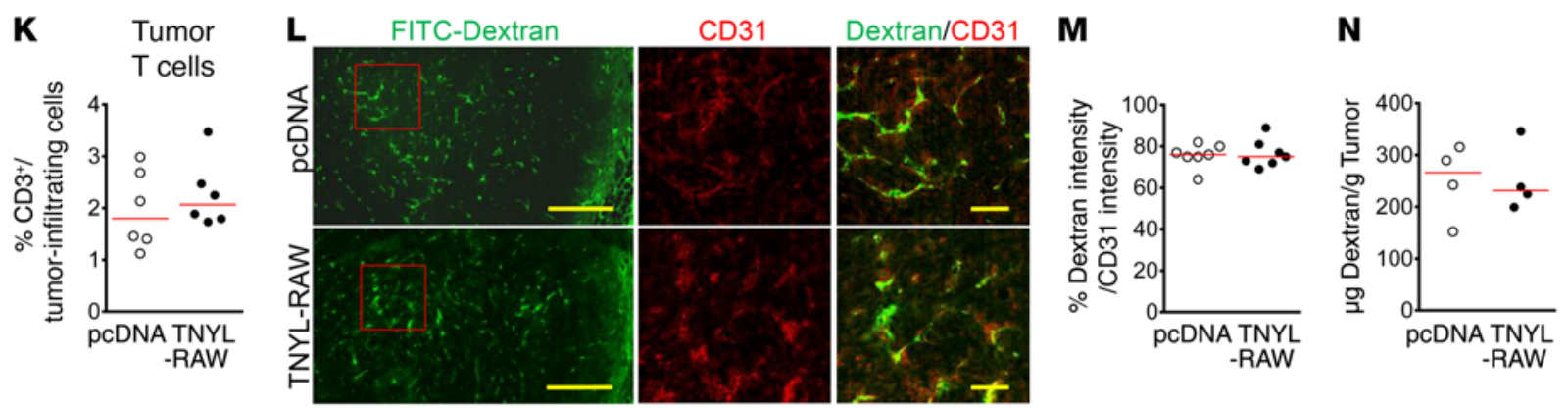

Figure 6. Ephrin B2/EPHB4 blockade inhibits 4T1 tumor growth, hematopoietic cell mobilization, and tumor infiltration with hematopoietic cells. (A) Schematic of experiment. On day 0, BALB/c mice were injected with pcDNA or TNYL-RAW vectors and with syngeneic, blue-Azurite ${ }^{+} 4 T 1$ cells; they were euthanized on day 7 for blood and tumor evaluation. (B) 4T1 tumor weight; median (red lines; $n=8 /$ group). (C) Plasma G-CSF levels in 4T1-bearing mice; median (red lines; $n=6$ /group). (D-F) wbc differential counts and colony-forming precursors in blood removed on day 7 from 4T1-bearing mice. Median (red lines; $n=6$ /group). (C) Azurite- methylcellulose colonies in 4T1 tumors; median (red lines; $n=6$ ); Azurite- colonies/10 $0^{7}$ Azurite ${ }^{+}$tumor cells. (H and I) Representative Gr-1 immunostaining of 4T1 tumor tissue from pcDNA-injected mouse. Scale bar: $20 \mu \mathrm{m}$ (H). Gr-1+ cell quantitation in 4T1 tumors (I); median \%

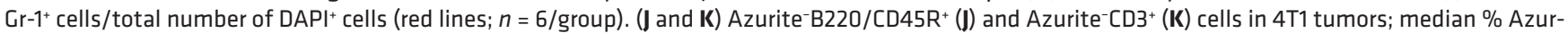
ite ${ }^{-}$infiltrating cells/Azurite ${ }^{+}$tumor cells $(n=6)$ by flow cytometry. (L-N) The tumor vasculature was visualized by FITC-dextran (green) perfusion and CD31 (red) immunostaining. (L) Representative confocal images of 4T1 tumors (day 7 after injection) from pcDNA- or TNYL-RAW-injected mice (scale bars: 500 $\mu \mathrm{m})$. The boxed areas are magnified on the right (scale bars: $100 \mu \mathrm{m}$ ). (M) Quantitation of FITC-dextran perfusion as a function of CD31; results expressed as \% fluorescence intensities; median (red lines, $n=7$ ). (N) Quantitation of FITC-dextran perfusion as a function of tumor weight. Ratio of $\mu g$ FITC-dextran to $g$ tumor weight; median (red lines, $n=4$ ). $P$ values by Student's $t$ test: ${ }^{* *} P<0.01$.

Consistent with this reduction in HSPCs mobilized to the blood, busulfan-treated recipients of blood from B11-treated mice displayed a significantly reduced number of donor-derived (tdTomato ${ }^{+}$HSPCs compared with controls, as reflected by flow

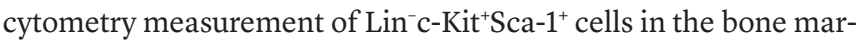
row (Figure 5E and Supplemental Figure 5D) and colony-forming precursors in blood after G-CSF mobilization on days 42 through 47 after transplant (Figure 5F and Supplemental Figure 5E). Bone marrows from recipients of B11 antibody-treated blood also displayed a significant reduction in donor-derived monocytes (Figure 5G and Supplemental Figure 5F) and neutrophils (Figure 5H and Supplemental Figure 5G) compared with controls.

To distinguish between the alternative possibilities that ephrin B2 signaling or EPHB4 signaling controls HSPCs mobilization in vivo, we took advantage of knockin $E f n b 2^{5 Y / 5 Y}$ mice, which have a tar- geted mutation of the 5 conserved tyrosines in the cytoplasmic tail, preventing phosphotyrosine-dependent ephrin $\mathrm{B} 2$ signaling (42); and Efnb2 $2^{\text {lacz//6YFAV }}$ mice, which lack all ephrin B2 signaling, as they express a protein that is both unable to become tyrosine phosphorylated and to interact with SH2- or PDZ-containing proteins, while retaining properly localized ephrin B2 on the cell surface (43). Compared with the littermate $E f n b 2^{W T / W T}$ controls, the ephrin B2 signaling-deficient mice displayed a normal mobilization response to G-CSF, as reflected by peripheral blood wbc counts and HSPC-derived colony counts (Figure 5, I and J). In sum, these experiments provide evidence that ephrin B2/EPHB4 interaction controls hematopoietic cell mobilization and that EPHB4 signaling mediates this regulation.

Blocking ephrin B2/EPHB4 reduces tumor growth. Since bone marrow-derived HSPCs and their progeny promote the growth of many tumor types by infiltrating tumor tissues and activating 
A

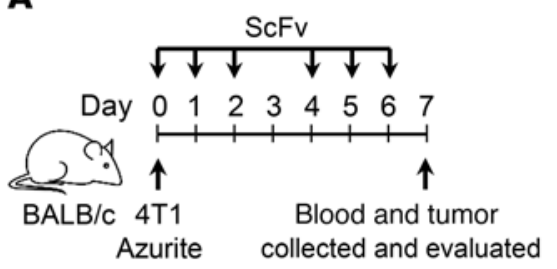

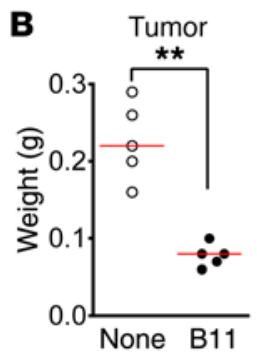
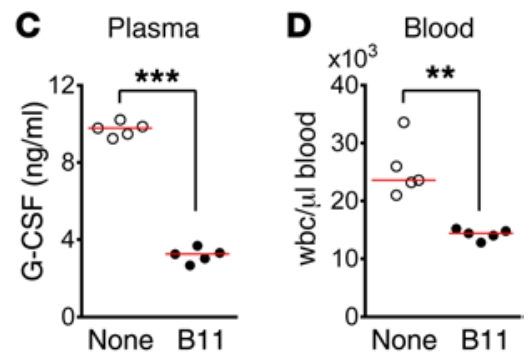
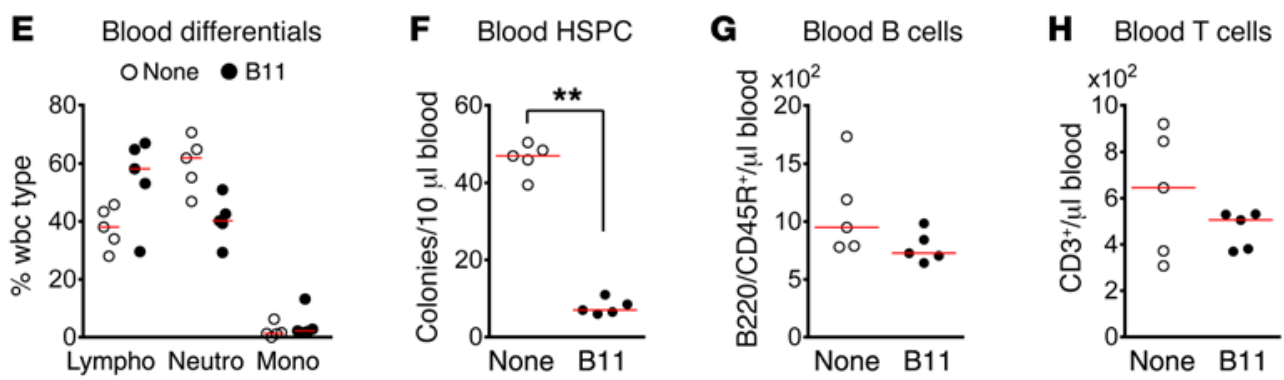

Figure 7. Ephrin B2 neutralization has antitumor activity. (A) Schematic of the experiment. BALB/c mice injected s.c. with Azurite ${ }^{+} 4 T 1$ cells on day 0 received 6 doses of scFv B11 antibody. On day 7 after 4 T1 cell injection, mice were bled and tumors removed. (B) 4T1 tumor weight in untreated and B11-treated mice; median (horizontal lines; $n=5$ /group). (C) Plasma G-CSF levels; median (horizontal lines; $n=5 /$ group). (D) Blood wbc; median (horizontal lines; $n=5$ /group). (E) Blood differential counts; median (horizontal lines; $n=5 /$ group). (F) Methylcellulose-supported colonies in blood; median (horizontal lines; $n=5$ /group). (G) B220/CD45R ${ }^{+}$B cells in blood; median (horizontal lines; $n=5 /$ group). (H) CD3 ${ }^{+}$cells in blood; median (horizontal lines; $n=5$ /group). $P$ values by Student's $t$ test: ${ }^{*} P<0.01,{ }^{* *} P<0.001$.

diverse pro-tumorigenic signals $(1-4,11)$, we tested whether ephrin B2/EPHB4 targeting to reduce HSPC mobilization reduces tumor growth. As proof of principle, we selected two mouse tumor models (4T1 mammary tumor and B16F10 melanoma) in which hematopoietic cell mobilization is an identified contributor to tumor progression; 4T1 tumors secrete active G-CSF $(12,44)$, and B16F10 secretes a mixture of less well-characterized mobilizing factors (45).

We tested the effects of TNYL-RAW blocking peptides in the $4 \mathrm{~T} 1$ tumor model (schematic of the experiment in Figure 6A; the 4T1 cells expressed the blue fluorescent protein Azurite and were injected into syngeneic WT BALB/c mice). When growing in mice expressing TNYL-RAW blocking peptides (plasma levels: $216 \pm 21$ $\mathrm{ng} / \mathrm{ml}$ on day 7), 4T1 cancers grew to a significantly lesser degree compared with tumors growing in control mice transduced with the pcDNA vector (Figure 6B and Supplemental Figure 6A, reflecting 4 independent experiments overall). Plasma levels of mouse G-CSF were lower in 4T1-bearing mice expressing TNYL-RAW compared with control (Figure 6C and Supplemental Figure 6B), likely reflecting the reduced $4 \mathrm{~T} 1$ tumor size and the direct correlation between 4T1 tumor size and circulating G-CSF levels (44). Consistent with the anti-mobilizing effects of TNYL-RAW expression after administration of exogenous G-CSF, TNYL-RAW expression in 4T1 tumor-bearing mice (endogenously secreting G-CSF) significantly reduced blood wbc compared with controls (Figure 6D and Supplemental Figure 6B), with a minimal change in the differential blood counts (Figure 6E and Supplemental Figure 6C) and the number of circulating HSPCs (Figure 6F and Supplemental Figure 6D). Notably, tumor-infiltrating HSPCs, distinguishable from the Azurite ${ }^{+}$tumor cells, were fewer in number in the TNYLRAW group compared with controls (Figure 6G and Supplemental
Figure 6E). Tumor-infiltrating Gr-1+ myeloid cells (Figure 6H) were also significantly reduced in the TNYL-RAW group compared with the control group (Figure 6I). This was attributable to a reduction in tumor-infiltrating neutrophils (Supplemental Figure 6E) in the TNYL-RAW group compared with controls, whereas the proportion of monocytes, B cells, and T cells was variable (Figure 6, J and K).

Since reduced blood perfusion can reduce tumor growth and EPHB4 neutralization was reported to reduce tumor vascularization in selected xenotransplants (41), we evaluated the effects of TNYL-RAW expression on blood perfusion of 4T1 tumors. FITC-dextran imaging showed that the $\mathrm{CD}^{+} 1^{+}$tumor vascular beds were similar in TNYL-RAW-expressing $(172 \pm 14.1 \mathrm{ng} / \mathrm{ml}$ plasma) and control mice (Figure 6L). In addition, quantitative analyses confirmed that tumor blood perfusion was similar in TNYL-RAW-expressing and control mice (Figure 6, M and N).

TNYL-RAW peptides did not reduce $4 \mathrm{~T} 1$ cell growth and survival in vitro, despite the presence of both ephrin B2 and EPHB4 in $4 \mathrm{~T} 1$ cells (Supplemental Figure 7, A-E), indicating that TNYLRAW treatment indirectly reduces $4 \mathrm{~T} 1$ tumor progression. Since we detected no measurable effect of TNYL-RAW treatment on 4T1 tumor blood perfusion, and previous evidence has linked bone marrow mobilization and hematopoietic cell infiltration to increased $4 \mathrm{~T} 1$ tumor progression $(4,44)$, we conclude that the current results support anticancer strategies that target the bone marrow to reduce mobilization of hematopoietic cells.

To confirm and strengthen these observations, we tested the effects of ephrin B2 neutralizing scFv antibody B11, which prevents ephrin B2 binding to EPHB4 (schematic of experiment in Figure 7A). B11 antibody significantly reduced 4T1 tumor growth (Figure 7B) associated with reduced plasma levels of endogenous G-CSF, 

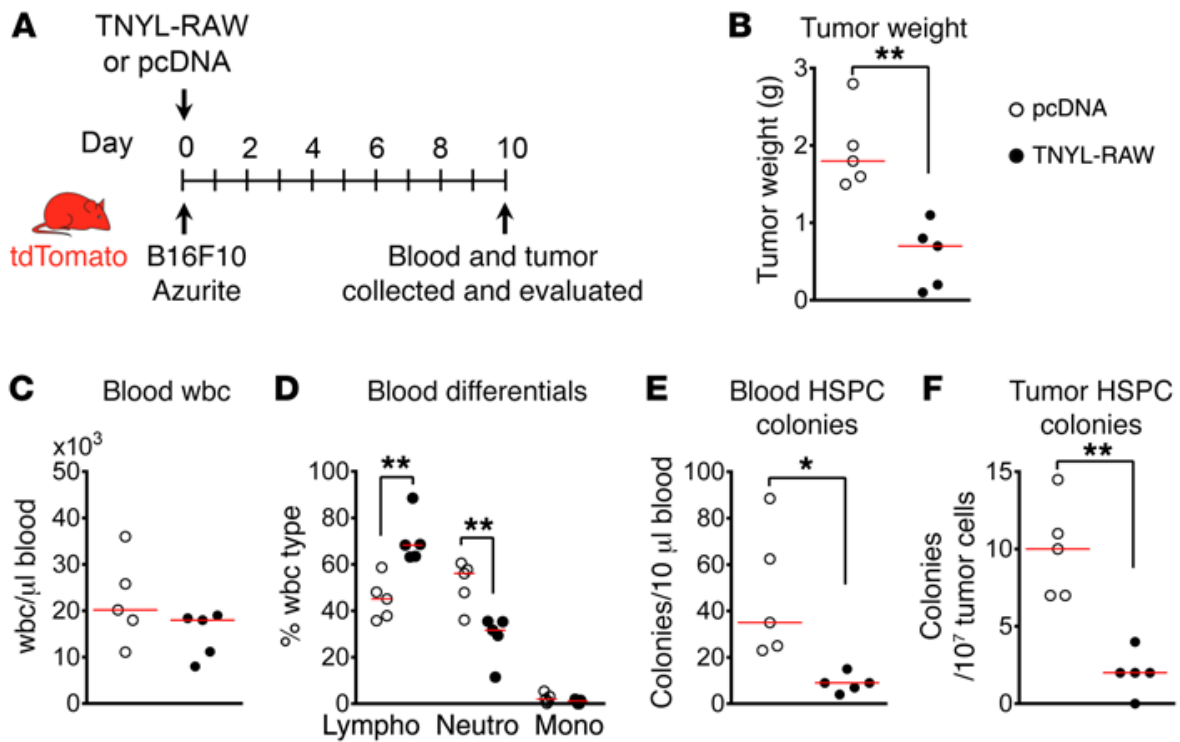

Figure 8. Ephrin B2/EPHB4 blockade reduces B16F10 tumor growth. (A) Schematic of experiment. Azurite ${ }^{+} \mathrm{B} 16 \mathrm{~F} 10$ cells were inoculated s.c. into syngeneic tdTomato mice transduced with TNYL-RAW or pcDNA vectors (day 0). On day 10 , mice were evaluated. (B-F) Evaluation of pcDNA and TNYL-RAW-transduced mice (day 10). (B) Tumor weight median (horizontal lines; $n=5 /$ group). (C) Blood wbc; median (horizontal lines; $n=5$ /group). (D) Differential blood counts; median (horizontal lines; $n=5 /$ group). (E) Colonies in blood; median (horizontal lines; $n=5$ / group). (F) Colonies (tdTomato ${ }^{+}$Azurite ${ }^{-}$) in single-cell-suspended tumors; median (horizontal lines; $n=5$ (group). $P$ values by Student's $t$ test: ${ }^{*} P<0.05,{ }^{*} P<0.01$ likely a reflection of the reduction in tumor burden (Figure 7C). The number of circulating wbc (Figure 7D) was significantly reduced in B11-treated mice, whereas the differential counts were not significantly altered (Figure 7E). Also reduced in B11-treated mice was the number of circulating colony-forming HSPCs (Figure 7F), but not the proportion of circulating B and T lymphocytes (Figure 7, G and H).

We extended our evaluation to the murine melanoma B16F10 tumor, which promotes HSPC mobilization through secretion of factors other than G-CSF (45) and lacks ephrin B2 (Supplemental Figure 7, A and B). Syngeneic tdTomato mice were injected with Azurite ${ }^{+}$B16F10 cells (schematic of experiment Figure 8A). In separate experiments, TNYL-RAW blocking peptides reduced B16F10 progression (Figure 8B and Supplemental Figure 8A). The blood profile of B16F10 tumor-bearing mice differed from that observed in 4T1 tumor-bearing mice: the number of circulating wbc was not significantly different in the TNYL-RAW and control groups (Figure 8C and Supplemental Figure 8B), and blood neutrophils were proportionally decreased in the TNYL-RAW group compared with control (Figure 8D and Supplemental Figure 8C); this was perhaps attributable to different mobilizing factors produced by the $4 \mathrm{~T} 1$ and B16F10 tumor cell lines $(45,46)$. Circulating $\mathrm{B}$ and $\mathrm{T}$ cells were similarly represented in the TNYL-RAW-treated B16F10 group compared with control (Supplemental Figure 8, D and E). Importantly, the TNYL-RAW-treated (180 $\pm 8.5 \mathrm{ng} /$ $\mathrm{ml}$ plasma) B16F10-bearing mice showed a significant reduction in the number of circulating (Figure 8E and Supplemental Figure $8 \mathrm{~F}$ ) and tumor-associated (Figure 8F and Supplemental Figure 8G) colony-forming HSPCs (their host derivation was based on tdTomato ${ }^{+}$Azurite status). $^{-}$

Analysis of individual colonies derived from tumor-infiltrating $\left(\right.$ tdTomato $\left.^{+}\right)$precursors identified $\mathrm{Gr}-1^{+}$Ter119- myeloid cells and Ter119+ Gr-1 $1^{-}$erythroid cells, consistent with a distinct progenitor origin (Figure 9A). Gr-1 immunostaining of B16F10 tumor tissues revealed scattered foci of infiltrating myeloid cells (Figure 9B). Flow cytometric analysis of tumor-infiltrating cell populations revealed that the proportion of tumor-associated neutrophils was significantly reduced in the TNYL-RAW group compared with control, whereas the proportion of monocytes, $\mathrm{B}$ cells, and T cells was similar in the TNYL-RAW and control groups (Figure 9, C and D, and Supplemental Figure 9, A-D). Confocal imaging additionally visualized very rare host (tdTomato ${ }^{+}$) $\mathrm{Lin}^{-} \mathrm{CD} 150^{+}$cells infiltrating the B16F10 tumor cells (Azurite ${ }^{+}$) in control (Figure 9E) but not TNYL-RAW-treated mice, confirming the presence of such cells in untreated B16F10 tumors. In addition, the relative numbers of tumor-associated $\mathrm{CD} 31^{+}$cells, a reflection of the tumor vasculature, were similar in $4 \mathrm{~T} 1$ tumors from B11-treated mice and controls, despite marked differences in tumor weight (Supplemental Figure 9E).

To test for a direct relationship between tumor-infiltrating HSPCs and increased tumor growth, we injected B16F10 tumor cells alone or with sorted td Tomato $^{+}$Lin $^{-}$CD $150^{+}$HSPCs into WT mice. Tumor weights were significantly greater when HSPCs were included in the tumor cell inoculum (Figure 9F). In addition, analysis of tumor-infiltrating tdTomato ${ }^{+}$cells $(1.3 \% \pm 0.01 \%$ of tumor cells) showed that myeloid $\mathrm{Ly} 6 \mathrm{C}^{+}$and $\mathrm{Ly} 6 \mathrm{G}^{+}$cells constituted the predominant infiltrating cell types (Figure 9G), indicating that the originally inoculated tdTomato ${ }^{+}$HSPCs had differentiated within the developing tumor. In aggregate, blocking ephrin B2/EPHB4 interaction reduces HSPC mobilization to the circulation and reduces tumor infiltration by HSPCs and myeloid lineage cells, which directly promote tumor growth.

\section{Discussion}

Increasingly, the bone marrow has emerged as an attractive target for therapies designed to abrogate pro-tumorigenic signals arising from HSPCs and their progeny (14). Yet no drugs are currently available to block the exit of HSPCs and other hematopoietic cells from the bone marrow to the circulation; this is attributable in part to an incomplete understanding of the complexities of this trafficking. Here, we provide insights into the biochemical regulation of hematopoietic cell trafficking from the bone marrow cavity to the circulation, describe successful approaches to block this process, and show examples of how this new strategy is effective in reducing experimental tumor growth. 
A

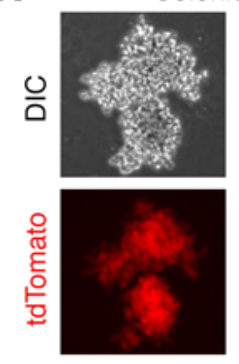

Colonies from B16F10 tumor
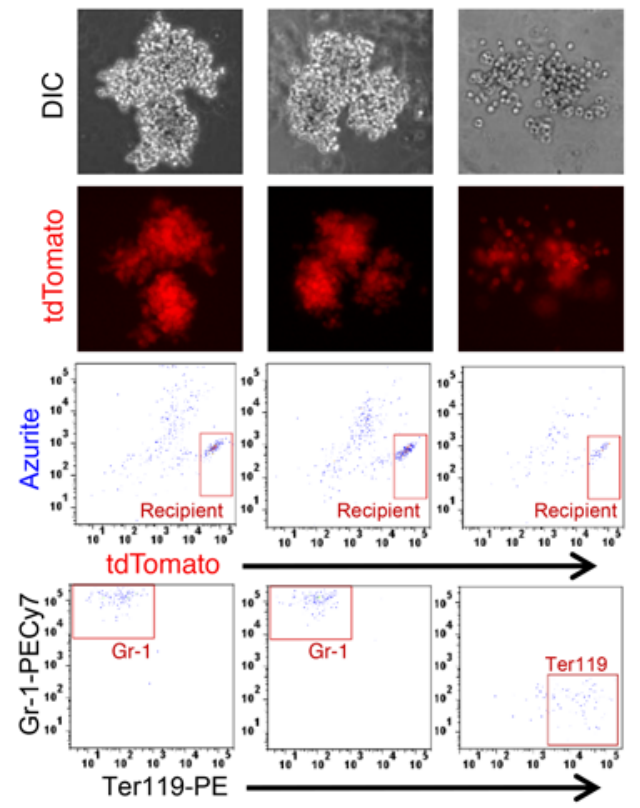

E

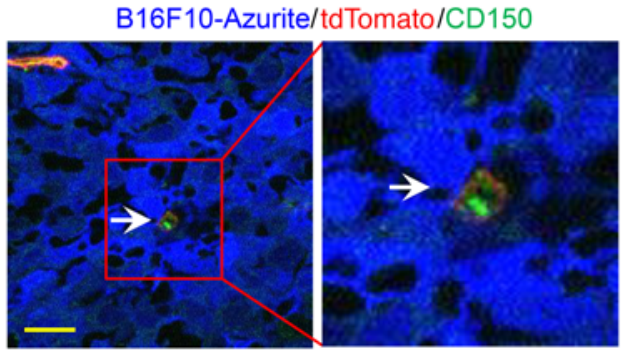

B

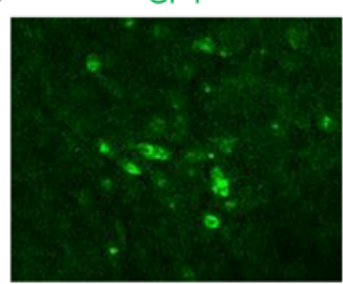

$\mathrm{Gr}-1$

Gr-1/B16F10-Azurite

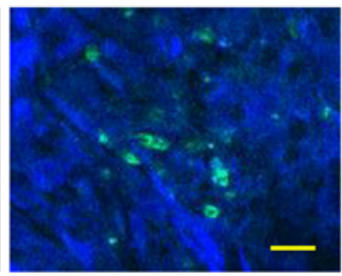

C Tumor monocytes

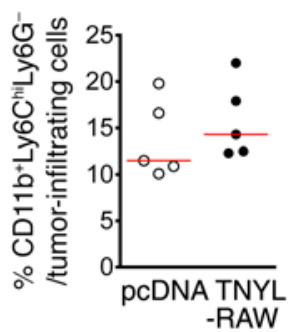

D

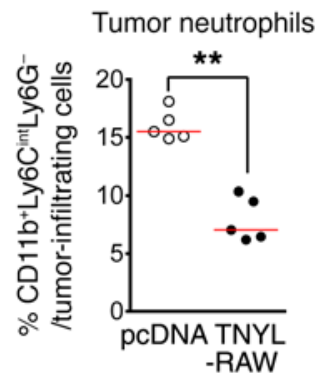

$\mathbf{F}$

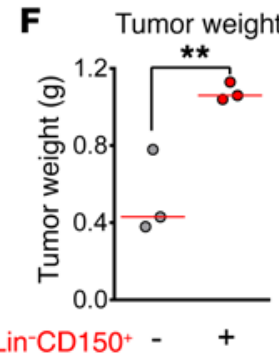

G

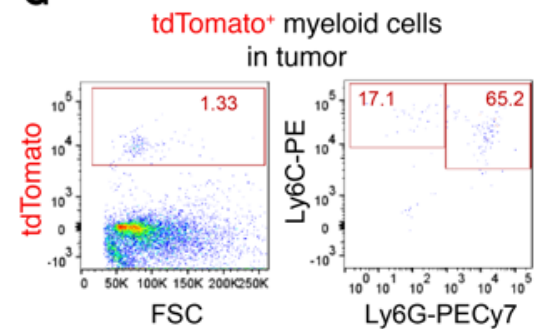

Figure 9. Ephrin B2/EPHB4 blockade reduces B16F10 tumor infiltration by HSPCs and myeloid cells. (A) Typical host-derived (tdTomato+Azurite-) colonies from tumor cell suspensions (pcDNA-transduced mice). DIC/fluorescence microscopy; representative flow cytometry profiles from tdTomato ${ }^{+}$Azurite individual colonies showing Gr-1+Ter119- and Ter119+Gr-1- cells. (B) Representative Gr-1 immunostaining of B16F10 tumor (pcDNA-transduced mouse). Scale bar: $20 \mu \mathrm{m}$. (C and D) Infiltration of tdTomato+Azurite- monocytes (C) and neutrophils (D); flow cytometry results from tumor cell suspensions; \% median (red lines; $n=5$ /group). (E) Confocal imaging of host (tdTomato+Azurite-) HSPCs (Lin-CD150+) in tumor (pcDNA-transduced mouse). Blue: Azurite ${ }^{+}$tumor cells. Red: a capillary-like structure and cell reflecting origin from the tdTomato mouse. Magenta: Lin immunostaining (not visible). Green: CD150+. Scale bar: $20 \mu \mathrm{m}$; $\times 2.5$ magnification (right panel). (F and G) WT mice were injected s.c. with syngeneic Azurite ${ }^{+}$B16F10 cells alone or mixed with sorted tdTomato ${ }^{+}$bone marrow-derived Lin-CD150+ HSPCs. (F) Tumor weight median (red line; $n=3 /$ group); (G) tdTomato ${ }^{+}$cells in a representative (of 3 ) tumors comprise

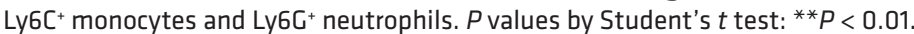

We made four important observations. First, we discovered the unambiguously complementary pattern of ephrin B2 and EPHB4 expression in the bone marrow, which is uncommon in other adult tissues $(47,48)$. Specifically, we established that the sinusoidal vessels express the tyrosine kinase EPHB4 receptor, but not its unique ligand, ephrin B2, or other EPH family receptors; and that the hematopoietic cells, most notably the HSPCs, express ephrin B2. This is important because sinusoidal vessels are a specialized vascular system that allows the trafficking of hematopoietic cells in and out of the bone marrow, and most HSPCs reside immediately adjacent to a sinusoidal vessel in the bone marrow, more so than other hematopoietic cells $(20,21)$. Since ephrin B2 and EPHB4 are transmembrane proteins (23), direct cell-to-cell contact is expected to restrict ephrin B2/EPHB4 signaling precisely at those sites where bone marrow HSPCs and sinusoidal endothelial cells come in contact.

The second observation we made links ephrin B2/EPHB4 signaling to functional control of HSPC exit from the bone mar- row by transendothelial migration, and unambiguously identifies EPHB4 forward signaling rather than ephrin B2 reverse signaling as critical to this function. Ephrin B2/EPHB4 signaling mediates cell functions such as adhesion and motility (23) that are essential to leukocyte transendothelial migration processes (49). Yet despite extensive investigation (50), there is no evidence for a functional role for B-type ephrins and EPHs in leukocyte transendothelial migration. Since ephrin B2 and EPHB4, respectively, mark arterial and venous endothelium in many tissues (51, 52), and circulating leukocyte subsets express these same molecules (53), a restricted role of ephrin B2/EPHB4 signaling in sustaining the transsinusoidal migration of hematopoietic cells in the bone marrow is striking.

The third, perhaps most important observation is that HSPCs exert direct control over their exit from the bone marrow cavity by initiating signaling interactions with the sinusoidal endothelial cells; this process can be effectively blocked. Thus, the results presented here challenge current thinking, according to which HSPCs are passive 
Table 1. List of antibodies used

\begin{tabular}{|c|c|c|c|}
\hline Antibody & Manufacturer & Clone no. & Cat. no. \\
\hline PE rat anti-mouse B220/CD45R & BD Pharmingen & RA3-6B2 & 553090 \\
\hline APC rat anti-mouse CD11b & BD Pharmingen & M1/70 & 101212 \\
\hline $\mathrm{PE} /$ Cy5 rat anti-mouse CD3 & BD Pharmingen & $9 \mathrm{H10}$ & 555278 \\
\hline Rat anti-mouse CD31 & BD Pharmingen & Mec13.3 & 553370 \\
\hline FITC rat anti-mouse CD31 & BioLegend & 390 & 102405 \\
\hline APC/Cy7 rat anti-mouse CD48 & BioLegend & HM48-1 & 103431 \\
\hline Biotin rat anti-mouse CD150 & eBioscience & $9 \mathrm{D1}$ & $13-1501-82$ \\
\hline PerCP/Cy5.5 anti-mouse CD150 & BioLegend & TC15-12F12.2 & 115922 \\
\hline FITC rat anti-mouse c-Kit & BD Pharmingen & 2B8 & 553354 \\
\hline PE rat anti-mouse c-Kit & BD Pharmingen & 2B8 & 553355 \\
\hline Rat anti-mouse Endomucin & Santa Cruz Biotechnology Inc. & V.7C7 & sc- 65495 \\
\hline Rabbit anti-mouse EPHA4 & Abcam & NA & ab126169 \\
\hline Rabbit anti-mouse EPHB1 & Santa Cruz Biotechnology Inc. & $\mathrm{H}-80$ & sc-28979 \\
\hline Goat anti-mouse EPHB2 & R\&D Systems & NA & AF467 \\
\hline Rabbit anti-mouse ephrin B2 & Abcam & NA & ab131536 \\
\hline Goat anti-mouse ephrin B2 & Abcam & NA & ab7043 \\
\hline Rabbit anti-human/mouse ephrin B2 & Santa Cruz Biotechnology Inc. & $\mathrm{N} / \mathrm{A}$ & sc-910 \\
\hline APC rat anti-mouse lineage & BD Pharmingen & NA & $51-9003632$ \\
\hline FITC rat anti-mouse Ly6C & BD Pharmingen & AL-21 & 553104 \\
\hline PE rat anti-mouse Ly6C & BD Pharmingen & AL-21 & 560592 \\
\hline FITC rat anti-mouse Ly6G & BD Pharmingen & $1 A 8$ & 551460 \\
\hline PE/Cy7 rat anti-mouse Ly6G & BioLegend & $1 \mathrm{~A} 8$ & 127618 \\
\hline Goat IgG anti-mouse EPHB4 & R\&D Systems & NA & AF446 \\
\hline Goat IgG anti-mouse EPHB4 & R\&D Systems & NA & AF3038 \\
\hline Rabbit anti-OPN & Abcam & NA & ab8448 \\
\hline Rabbit anti-mouse Sp7 & Abcam & NA & $a b 22552$ \\
\hline Biotin rat anti-mouse Sca-1 & BioLegend & E13-161.7 & 1225074 \\
\hline FITC rat anti-mouse Sca-1 & BD Pharmingen & E13-161.7 & 553335 \\
\hline $\mathrm{PE} /$ Cy7 rat anti-mouse Sca-1 & BioLegend & E13-161.7 & 122514 \\
\hline PE/Cy5 rat anti-mouse Ter119 & BioLegend & TER-119 & 116210 \\
\hline PE rat anti-mouse VEGFR3 & Miltenyi Biotec & AFL4 & $130-102-216$ \\
\hline Mouse anti-actin-HRP & Santa Cruz Biotechnology Inc. & $\lceil 4$ & sc-47778HRP \\
\hline Coat anti-human Fc-HRP & Southern Biotech & NA & 2048-05 \\
\hline Rabbit polyclonal anti-CAPDH & Santa Cruz Biotechnology Inc. & NA & sc- 25778 \\
\hline NA, not applicable. & & & \\
\hline
\end{tabular}

bystanders during bone marrow mobilization, swept out of the bone marrow by a process orchestrated by mature myeloid cells $(6,16,17)$. This view is mainly built on observations from the use of G-CSF, the most potent bone marrow HSPC mobilizing agent currently available (6), and recognition that G-CSF does not mobilize HSPCs acting directly on these cells but rather through myeloid cells $(16,19)$. In this context, G-CSF would disrupt retentive signals imposed upon HSPCs by the microenvironment of the bone marrow niche in which they reside, acting indirectly through mechanisms that remain poorly defined (6). The current results point to a facilitating role for G-CSF in bringing HSPCs in closer proximity to the sinusoidal endothelium, identifying instead ephrin B2/EPHB4 signaling as a limiting step in HSPC mobilization from the bone marrow to the blood.

The fourth observation has important therapeutic implications for reducing damaging effects from mobilized HSPCs, as seen in ischemic diseases such as myocardial infarction and cancer $(4,10,14)$. The current results demonstrate that reducing bone marrow mobilization of HSPCs and other ephrin $\mathrm{B}^{+}$ myeloid populations reduces the rate of tumor growth. There is overwhelming evidence directly linking the presence of various HSPCs and committed myeloid cell populations in blood and tumor microenvironments with accelerated tumor progression and risk for tumor metastasis $(1-5,11)$. HSPCs are abnormally elevated in blood, tumor, and other tissues of tumor-bearing mice and humans, and differentiate into pro-tumorigenic myeloid cells in response to tumor-derived factors $(4,12,13)$. Thus, a strategy that effectively limits the mobilization of HSPCs and other myeloid cell populations would address an emerging need.

We have identified ephrin B2/EPHB4-dependent hematopoietic cell exit from the bone marrow as a pathway that sustains supply of pro-tumorigenic hematopoietic cells. Ephrin B2-neutralizing antibodies (54), EPHB4-neutralizing antibodies, drugs that prevent ephrin $\mathrm{B} 2 / \mathrm{EPHB} 4$ binding, and EPHB4 signaling inhibitors can be effective in blocking this pathway and offer hope for a new anticancer strategy.

\section{Methods}

Cells and cell culture. The mouse pancreatic islet endothelial cell line MS-1 (ATCC) was cultured on gelatin-coated plates in high-glucose DMEM (Invitrogen) with $5 \%$ FBS (Sigma-Aldrich). The mouse bone marrow stromal cell line MS- 5 (Creative Bioarray), the mouse melanoma cell line B16F10 (ATCC), and the mouse lung carcinoma LLC1 (ATCC) were cultured in high-glucose DMEM with 10\% FBS. The 4 T1 mouse mammary tumor cell line (ATCC) was cultured in RPMI-1640 with 10\% FBS. The myristoylated AKT mouse BMECs (30) were cultured on fibronectincoated plates (Sigma-Aldrich, F0895) in low-glucose DMEM (Invitrogen), with 50\% Ham F-12 media, nonessential amino acids, $20 \mathrm{mM}$ HEPES, $100 \mu \mathrm{g} / \mathrm{ml}$ heparin, 20\% FBS, and $50 \mu \mathrm{g} / \mathrm{ml}$ endothelial cell growth supplement (Sigma-Aldrich). Primary calvarial osteoblasts (a gift of K. Holmbeck, National Institute of Dental and Craniofacial Research [NIDCR]/NIH Bethesda, Maryland, USA) were isolated as described in ref. 55. Isolation of primary bone marrow sinusoidal endothelial cells is described in Supplemental Methods.

Bone marrow isolation and enrichment. Bone marrow was harvested by crushing mouse femurs, tibiae, and hips. After red cell lysis, lineage depletion was performed using a Mouse Hematopoietic Progenitor Cell Isolation Kit (STEMCELL Technologies). Briefly, bone marrow cells incubated first with biotin-conjugated antibodies and subsequently with streptavidin-coated magnetic particles were separated using an EasySep magnet. Lineage-negative cells were recovered. All antibodies used in this study are listed in Table 1.

Transendothelial migration, endothelial permeability, and adhesion. MS- 1 cells or BMECs $\left(1 \times 10^{4}\right)$ were used to generate a monolayer separating the chambers of HTS Transwell 96-well plates with 5- $\mu \mathrm{m}$-poresize polycarbonate membrane (Corning), as described previously (56). 
After 24 hours, bone marrow mononuclear cells $\left(1 \times 10^{6}\right)$ suspended in $50 \mu$ l MethoCult M3231 (STEMCELL Technologies) were added onto the monolayer in the top chamber of Transwells; the bottom chamber was filled with $200 \mu \mathrm{l}$ IMDM with $10 \%$ FBS. The integrity of the endothelial monolayer (used for transmigration) was evaluated with 1 - $\mu$ m-diameter fluorescent beads (FluoSpheres, Molecular Probes) added to the upper compartment. After 14 hours, the transmigrated cells were collected, counted, and evaluated by flow cytometry. Endothelial monolayer permeability and adhesion assays are described in Supplemental Methods.

Immunoprecipitation and immunoblotting. Immunoprecipitation, performed using Dynabeads Protein G Immunoprecipitation Kit (ThermoFisher Scientific), is described in Supplemental Methods. Immunoblotting, performed as described previously (57), is detailed in Supplemental Methods. Full uncut gels are shown in Supplemental Figure 10.

Colony-forming assays, cell proliferation, cell death. Peripheral blood $(10 \mu \mathrm{l})$ or bone marrow cells $\left(1 \times 10^{4}\right)$ were mixed into $1.5 \mathrm{ml}$ MethoCult 3434 medium (STEMCELL Technologies) and plated into wells (6-well plates). Colonies were scored at day 10 by an observer without knowledge of the experimental design. Cell proliferation and cell death assays are described in Supplemental Methods.

ELISAs. Levels of TNYL-RAW-Fc were measured by an indirect ELISA; dilutions of plasma were added to 96-well plates precoated with human IgG-Fc (10 $\mu \mathrm{g} / \mathrm{ml}$ in PBS, Life Technologies). After incubation (1 hour) and washes (0.05\% Tween 20/PBS), goat anti-human Fc-HRP (Southern Biotech, 1:700) was added (1 hour). Recombinant human Fc (R\&D Systems) was used as a standard. After washes, TMB substrate (Thermo Scientific) was added and incubated until the desired color intensity was reached. The reaction was stopped (sulfuric acid) and absorbance measured at $450 \mathrm{~nm}$ using a BMG FLUOstar OPTIMA Microplate Reader. Plasma G-CSF levels were measured by ELISA (R\&D Systems) as detailed in Supplemental Methods.

Plasmids. TNYL-RAW fused with human Fc (TNYL-RAW-Fc) (58), EPHB4 WT, and EPHB4 K647R mutant plasmids (59) were a gift of E. Pasquale, Sanford Burnham Institute, La Jolla, California, USA. The EPHB4 and ЕPHB4 K647R plasmids were subcloned into pEntr4 vector (Addgene plasmid 19364), using EcoR I restriction enzyme and subsequently subjected to LR Clonase IImediated (Invitrogen) recombination using pLenti-PGK-puroDEST plasmid (Addgene plasmid 19068). Third-generation lentiviral shRNA constructs targeting mEPHB4 (TRCN0000023619: CCGGCGGATCTGAAATGGGTGACTTCTCGAGAAGTCACCCATTTCAGATCCGTTTTT; and TRCNO000023621: CCGGCGGTTATGATCCTCACGGAATCTCGAGATTCCGTGAGGATCATAACCGTTTTT) were from Sigma-Aldrich. pLV-Azurite was from Addgene (plasmid \#36086).

Gene silencing and expression. Lentiviruses for Azurite, $\mathrm{MEPHB} 4$ shRNA, EPHB4, or EPHB4 K647R were produced in 293T cells (ATCC) by transfection of a transducing vector with third-generation packaging system plasmids (pMDLg/pRRE; pRSV-Rev, and a VSVG envelope plasmid). Virus-containing supernatant was collected 72 hours after transfection. RNA extracted using an RNeasy Micro Kit (QIAGEN 74004) was used to synthesize cDNA with a High-Capacity cDNA Reverse Transcription Kit (Applied Biosystems 4368814) according to the manufacturer's instructions. Relative mRNA expression was measured by real-time PCR, as described in Supplemental Methods.
Flow cytometry. Cells were stained on ice for 30 minutes with directly labeled antibodies; DAPI ( $50 \mu \mathrm{g} / \mathrm{ml}$; Invitrogen) was used for cell viability. Cells were analyzed on an LSR II or LSR Fortessa (BD) instrument. Sample acquisition was carried out with FACSDiva software (BD, version 8.0.1); results were analyzed and displayed using FlowJo software (version 7.6.5).

Mice, G-CSF mobilization, AMD3100 mobilization, and hydrodynamic injection. The Efnb $2^{5 \mathrm{Y} / 5 Y}$ and $E f n b 2^{W T / W T}$ mice $(42,57)$ were a gift from A. Acker-Palmer (Gutenberg University, Mainz, Germany), and the Efnb2 $2^{\text {lacZ/6YFAV }}$ (43) and Efnb2 ${ }^{\text {H2BGFP }}$ reporter mice (27) were gifts from P. Soriano (Mount Sinai Health System, New York, New York, USA) and R. Wong (UCSF, San Francisco, California, USA). The

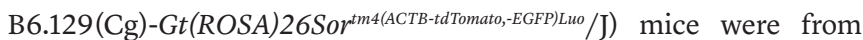
the Jackson Laboratory. G-CSF-induced mobilization was performed as described previously (16). AMD3100-induced mobilization was induced as described previously $(28,38)$. Hydrodynamic injection of plasmid DNA was performed as described previously (35). Briefly, 20 $\mu \mathrm{g}$ TNYL-RAW-Fc plasmid or pcDNA3 vector in $2 \mathrm{ml} 0.9 \% \mathrm{NaCl}$ was injected into the tail vein of mice within 6 seconds.

Ephrin B2 scFv antibody treatment. Anti-ephrin B2 scFv antibodies B11 and 2B1 were previously described (41). Mice were injected i.v. with $\mathrm{B} 11$ or $2 \mathrm{~B} 1$ ( $4 \mathrm{mg} / \mathrm{kg}$ in $0.2 \mathrm{ml} \mathrm{PBS}$ ) until reaching a total dose of $20 \mathrm{mg} / \mathrm{kg}$ or $24 \mathrm{mg} / \mathrm{kg}$, as illustrated in Figure 5A or Figure 7A. Control animals received vehicle (PBS) alone.

Transplantation, homing, and engraftment. Donor tdTomato mice were mobilized with G-CSF (day -5 to day -1 ). Recipient C57BL/6 mice were injected i.p. with busulfan $(0.1 \mathrm{ml} / 10 \mathrm{~g}$ body weight). Fractionated busulfan $(4 \times 25 \mathrm{mg} / \mathrm{kg}$ ) was administered (day -4 to day -1$)$, followed by transplantation at day 0 , as described previously (36). Heparinized blood $(200 \mu \mathrm{l})$ collected from donor tdTomato mice was immediately injected into the tail vein of recipient mice on day 0. Protocols for hematopoietic cell homing and engraftment are described in Supplemental Methods.

Mouse tumor models. 4T1 mouse mammary tumor cells $(2 \times$ $\left.10^{6}\right)$ and B16F10 mouse melanoma cells $\left(5 \times 10^{6}\right)$ transduced with pLV-Azurite were injected s.c. into syngeneic BALB/c mice (4T1) and tdTomato mice (B16F10). For TNYL-RAW transduction in vivo, the mice received hydrodynamic injection of $20 \mu \mathrm{g} / 2 \mathrm{ml}$ pcDNA or TNYL-RAW plasmids through the tail vein on the same day as tumor cell injection. For scFv ephrin B2 antibody treatment, BALB/c mice were injected i.v. with $4 \mathrm{mg} / \mathrm{kg}$ antibody starting on the same day as 4T1-Azurite cell injection; antibody was injected until a total dose of $24 \mathrm{mg} / \mathrm{kg}$ was reached (Figure 6A). Ten (B16F10) or 7 days (4T1) after cell injection, mice were sacrificed for further analysis. Coinjection of B16F10 tumor cells and HSPCs is described in Supplemental Methods.

Bone marrow clarification. Hydrogel solution was prepared as described previously (60) by mixing $4 \mathrm{ml}$ acrylamide (40\%), $1 \mathrm{ml}$ bis-acrylamide (2\%), $100 \mathrm{mg}$ VA-044 initiator (10\% wt), $4 \mathrm{ml}$ of $10 \times \mathrm{PBS}, 10 \mathrm{ml}$ of

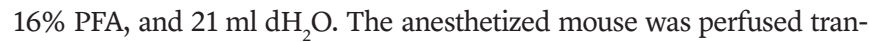
scardially with $20 \mathrm{ml}$ of ice-cold $1 \times \mathrm{PBS}$, followed by perfusion with hydrogel solution (10 ml per minute). Femurs were collected in tubes containing $5 \mathrm{ml}$ hydrogel solution and incubated $\left(24\right.$ hours, $\left.4^{\circ} \mathrm{C}\right)$. The stained bone marrow was cleared in RapidClear 1.49 solution (SunJin Lab).

Immunofluorescence and confocal microscopy. Tissue samples were permeabilized in $0.5 \%$ Triton X-100/PBS (15 minutes), washed in $1 \times$ PBS, and incubated ( 2 hours) with blocking solution (2\% BSA, 10\% donkey serum, and $0.1 \%$ Triton X-100/PBS). Samples were rinsed (PBS) and incubated first with primary antibodies (1:100 dilution; $4^{\circ} \mathrm{C}$ overnight); washed 
(0.1\% Triton X-100/PBS, 3 times, 30 minutes); and then incubated with fluorescent secondary antibodies (1:300 dilution, 2 hours). After washing (3 times, 30 minutes in 0.1\% Triton X-100/PBS), glass coverslips were mounted onto the slides, and sealed. Confocal images were acquired with Zen 2012 software on a Zeiss LSM 710 or 780 confocal system. Threedimensional reconstructions of Z-stack images were obtained using Amira software (version 6.0). ImageJ software (NIH) was utilized to quantify the infiltrating cells in tumor tissues. Percentages of positive cells/total DAPI ${ }^{+}$ cells were calculated by counting 10 randomly chosen fields from confocal images. At least 1,000 DAPI ${ }^{+}$cells were counted.

Intravital imaging. Intravital live imaging of skull bone marrow was carried out with minor modifications of published protocols (29). Lin-Sca- $1^{+}$cells were selected from the bone marrow of tdTomato mice and injected into the tail vein of C57BL/6 mice. The recipient mice were then mobilized with G-CSF for 5 days. Mice were anesthetized with a cocktail of $80 \mathrm{mg} / \mathrm{kg}$ ketamine and $10 \mathrm{mg} / \mathrm{kg}$ xylazine and immobilized on a custom-made stage. For visualization of blood vessels in vivo, mice were injected retro-orbitally with $4 \mathrm{mg}$ FITC-conjugated 2,000-kDa dextran (Sigma-Aldrich) in $200 \mu \mathrm{l}$ saline. The skull bone marrow was exposed by the removal of a small area of skin, and the head of the animal was immobilized in a custom-made holder. Imaging was performed using an inverted confocal Olympus FV1000 equipped with a $25 \times$ water lens (XL Plan N 1.05 N.A.). The exposed skull bone was bathed with saline and covered with a coverslip. Intravital time-lapse images were acquired by exciting the specimen with $488 \mathrm{~nm}$ (FITC-dextran) and $561 \mathrm{~nm}$ (tdTomato) lasers. Laser power was kept below $1 \%$ to minimize photo damage. Z-stacks (5 sections, optical slice $1 \mu \mathrm{m}$ ) were acquired every 6 seconds. Volume rendering was performed using Imaris (Bitplane). Analysis of bone marrow sinusoidal vessel leakage is described in Supplemental Methods.

Statistics. Data are expressed as mean \pm SD. An unpaired 2-tailed Student $t$ test was used for statistical analysis of differences between two groups. One-way ANOVA with Dunnett's multiple comparison test was used for statistical analysis of differences comparing three or more groups. A $P$ value less than 0.05 was considered significant.

Study approval. All animal studies were approved by the Institutional Animal Care and Use Committee of the CCR, National Cancer Institute (NCI), NIH. The studies were conducted in adherence to the NIH Guide for the Care and Use of Laboratory Animals (National Academies Press, 2011).

\section{Author contributions}

HK and GT conceived of the study, generated the hypothesis, and designed and evaluated experiments; HK performed the experiments with assistance from GT and OS and performed statistical analyses; RW performed intravital microscopy imaging; $\mathrm{MH}$, JMB, MGP, and JLMT provided essential reagents and critically reviewed the manuscript; GT and HK wrote the manuscript.

\section{Acknowledgments}

We thank E. Pasquale for constructs; D. Soriano, R. Wong, and A. Acker-Palmer for mice; CCR animal facility personnel; D. Lowy and members of the Laboratory of Cellular Oncology for help in various aspects of this work. This work was supported by the intramural research program of the CCR/NCI/NIH. JLMT is supported by the Regional Government of Madrid (Angiobodies Programme BIPEDD2 S2011/BMD-2312) and the European Social Fund; JMB is supported by the Ansary Stem Cell Institute, an American Society of Hematology Scholar Award, an American Federation for Aging Research grant, and a Leukemia and Lymphoma Society Quest for Cures award.

Address correspondence to: Giovanna Tosato, Laboratory of Cellular Oncology, 37 Convent Drive, NIH Bethesda Campus, Building 37, Room 4124, Bethesda, Maryland 20892, USA. Phone: 301.594.9596; E-mail: Tosatog@mail.nih.gov.
1. Mantovani A, Allavena P, Sica A, Balkwill F. Cancer-related inflammation. Nature. 2008;454(7203):436-444.

2. Psaila B, Lyden D. The metastatic niche: adapting the foreign soil. Nat Rev Cancer. 2009;9(4):285-293.

3. Hanahan D, Coussens LM. Accessories to the crime: functions of cells recruited to the tumor microenvironment. Cancer Cell. 2012;21(3):309-322.

4. Talmadge JE, Gabrilovich DI. History of myeloid-derived suppressor cells. Nat Rev Cancer. 2013;13(10):739-752.

5. Wculek SK, Malanchi I. Neutrophils support lung colonization of metastasis-initiating breast cancer cells. Nature. 2015;528(7582):413-417.

6. Bendall LJ, Bradstock KF. G-CSF: from granulopoietic stimulant to bone marrow stem cell mobilizing agent. Cytokine Growth Factor Rev. 2014;25(4):355-367.

7. Scadden DT. The stem-cell niche as an entity of action. Nature. 2006;441(7097):1075-1079.

8. Wright DE, Wagers AJ, Gulati AP, Johnson FL, Weissman IL. Physiological migration of hematopoietic stem and progenitor cells. Science. 2001;294(5548):1933-1936.

9. Massberg S, et al. Immunosurveillance by hematopoietic progenitor cells trafficking through blood, lymph, and peripheral tissues. Cell.
2007;131(5):994-1008.

10. Dutta $P$, et al. Myocardial infarction accelerates atherosclerosis. Nature. 2012;487(7407):325-329.

11. Giles AJ, et al. Activation of Hematopoietic Stem/ Progenitor Cells Promotes Immunosuppression Within the Pre-metastatic Niche. Cancer Res. 2016;76(6):1335-1347.

12. DuPre' SA, Hunter KW. Murine mammary carcinoma 4T1 induces a leukemoid reaction with splenomegaly: association with tumor-derived growth factors. Exp Mol Pathol. 2007;82(1):12-24.

13. Younos IH, Dafferner AJ, Gulen D, Britton HC, Talmadge JE. Tumor regulation of myeloidderived suppressor cell proliferation and trafficking. Int Immunopharmacol. 2012;13(3):245-256.

14. Wagers AJ. The stem cell niche in regenerative medicine. Cell Stem Cell. 2012;10(4):362-369.

15. Hopman RK, DiPersio JF. Advances in stem cell mobilization. Blood Rev. 2014;28(1):31-40.

16. Christopher MJ, Rao M, Liu F, Woloszynek JR, Link DC. Expression of the G-CSF receptor in monocytic cells is sufficient to mediate hematopoietic progenitor mobilization by G-CSF in mice. J Exp Med. 2011;208(2):251-260.

17. Chow A, et al. Bone marrow CD169+ macrophages promote the retention of hematopoietic stem and progenitor cells in the mesenchymal stem cell niche. JExp Med. 2011;208(2):261-271.

18. Lévesque JP, Hendy J, Takamatsu Y, Simmons PJ, Bendall LJ. Disruption of the CXCR4/CXCL12 chemotactic interaction during hematopoietic stem cell mobilization induced by GCSF or cyclophosphamide. J Clin Invest. 2003;111(2):187-196.

19. Pelus LM, Bian H, King AG, Fukuda S. Neutrophil-derived MMP-9 mediates synergistic mobilization of hematopoietic stem and progenitor cells by the combination of G-CSF and the chemokines GRObeta/CXCL2 and GRObetaT/ CXCL2delta4. Blood. 2004;103(1):110-119.

20. Kunisaki Y, et al. Arteriolar niches maintain haematopoietic stem cell quiescence. Nature. 2013;502(7473):637-643.

21. Morrison SJ, Scadden DT. The bone marrow niche for haematopoietic stem cells. Nature. 2014;505(7483):327-334.

22. Nombela-Arrieta C, et al. Quantitative imaging of haematopoietic stem and progenitor cell localization and hypoxic status in the bone marrow microenvironment. Nat Cell Biol. 2013;15(5):533-543.

23. Pasquale EB. Eph-ephrin bidirectional signaling in physiology and disease. Cell. 2008;133(1):38-52.

24. Kusumbe AP, Ramasamy SK, Adams RH. Coupling of angiogenesis and osteogenesis by a specific vessel subtype in bone. Nature. 2014;507(7492):323-328. 
25. Pennisi A, et al. The ephrinB2/EphB4 axis is dysregulated in osteoprogenitors from myeloma patients and its activation affects myeloma bone disease and tumor growth. Blood. 2009;114(9):1803-1812.

26. Zhao C, et al. Bidirectional ephrinB2-EphB4 signaling controls bone homeostasis. Cell Metab. 2006;4(2):111-121.

27. Davy A, Soriano P. Ephrin-B2 forward signaling regulates somite patterning and neural crest cell development. Dev Biol. 2007;304(1):182-193.

28. Itkin T, et al. Distinct bone marrow blood vessels differentially regulate haematopoiesis. Nature. 2016;532(7599):323-328.

29. Lo Celso C, Lin CP, Scadden DT. In vivo imaging of transplanted hematopoietic stem and progenitor cells in mouse calvarium bone marrow. Nat Protoc. 2011;6(1):1-14.

30. Poulos MG, et al. Vascular platform to define hematopoietic stem cell factors and enhance regenerative hematopoiesis. Stem Cell Reports. 2015;5(5):881-894.

31. Yang NY, Pasquale EB, Owen LB, Ethell IM. The EphB4 receptor-tyrosine kinase promotes the migration of melanoma cells through Rhomediated actin cytoskeleton reorganization. J Biol Chem. 2006;281(43):32574-32586.

32. Pasquale EB. Eph receptor signalling casts a wide net on cell behaviour. Nat Rev Mol Cell Biol. 2005;6(6):462-475.

33. Muller WA. The regulation of transendothelial migration: new knowledge and new questions. Cardiovasc Res. 2015;107(3):310-320.

34. Koolpe M, Burgess R, Dail M, Pasquale EB. EphB receptor-binding peptides identified by phage display enable design of an antagonist with ephrin-like affinity. J Biol Chem. 2005;280(17):17301-17311.

35. Huang LR, Wu HL, Chen PJ, Chen DS. An immunocompetent mouse model for the tolerance of human chronic hepatitis B virus infection. Proc Natl Acad Sci U S A. 2006;103(47):17862-17867.

36. Westerhof GR, et al. Comparison of different busulfan analogues for depletion of hematopoietic stem cells and promotion of donor-type chimerism in murine bone marrow transplant recipients. Cancer Res. 2000;60(19):5470-5478. 37. Lévesque JP, Hendy J, Winkler IG, Takamatsu
Y, Simmons PJ. Granulocyte colony-stimulating factor induces the release in the bone marrow of proteases that cleave c-KIT receptor (CD117) from the surface of hematopoietic progenitor cells. Exp Hematol. 2003;31(2):109-117.

38. Winkler IG, et al. Hematopoietic stem cell mobilizing agents G-CSF, cyclophosphamide or AMD3100 have distinct mechanisms of action on bone marrow HSC niches and bone formation. Leukemia. 2012;26(7):1594-1601.

39. Nguyen TM, et al. EphB4 expressing stromal cells exhibit an enhanced capacity for hematopoietic stem cell maintenance. Stem Cells. 2015;33(9):2838-2849.

40. Lévesque JP, Helwani FM, Winkler IG. The endosteal 'osteoblastic' niche and its role in hematopoietic stem cell homing and mobilization. Leukemia. 2010;24(12):1979-1992.

41. Abéngozar MA, et al. Blocking ephrinB2 with highly specific antibodies inhibits angiogenesis, lymphangiogenesis, and tumor growth. Blood. 2012;119(19):4565-4576.

42. Mäkinen T, et al. PDZ interaction site in ephrinB2 is required for the remodeling of lymphatic vasculature. Genes Dev. 2005;19(3):397-410.

43. Thakar S, Chenaux G, Henkemeyer M. Critical roles for EphB and ephrin-B bidirectional signalling in retinocollicular mapping. Nat Commun. 2011;2:431.

44. Waight JD, Hu Q, Miller A, Liu S, Abrams SI. Tumor-derived G-CSF facilitates neoplastic growth through a granulocytic myeloid-derived suppressor cell-dependent mechanism. PLoS One. 2011;6(11):e27690.

45. Kaplan RN, et al. VEGFR1-positive haematopoietic bone marrow progenitors initiate the pre-metastatic niche. Nature. 2005;438(7069):820-827.

46. Ohnuki H, et al. Tumor-infiltrating myeloid cells activate Dll4/Notch/TGF- $\beta$ signaling to drive malignant progression. Cancer Res. 2014;74(7):2038-2049.

47. Munarini N, et al. Altered mammary epithelial development, pattern formation and involution in transgenic mice expressing the EphB4 receptor tyrosine kinase. J Cell Sci. 2002;115(Pt 1):25-37.

48. Genander M, Frisén J. Ephrins and Eph receptors in stem cells and cancer. Curr Opin Cell Biol.
2010;22(5):611-616.

49. Butcher EC. Leukocyte-endothelial cell recognition: three (or more) steps to specificity and diversity. Cell.1991;67(6):1033-1036.

50. Kreuger J, Phillipson M. Targeting vascular and leukocyte communication in angiogenesis, inflammation and fibrosis. Nat Rev Drug Discov. 2016;15(2):125-142.

51. Wang HU, Chen ZF, Anderson DJ. Molecular distinction and angiogenic interaction between embryonic arteries and veins revealed by ephrin-B2 and its receptor Eph-B4. Cell.1998;93(5):741-753.

52. Adams RH, et al. Roles of ephrinB ligands and EphB receptors in cardiovascular development: demarcation of arterial/venous domains, vascular morphogenesis, and sprouting angiogenesis. Genes Dev. 1999;13(3):295-306.

53. Ivanov AI, Romanovsky AA. Putative dual role of ephrin-Eph receptor interactions in inflammation. IUBMB Life. 2006;58(7):389-394.

54. Zhang G, Brady J, Liang WC, Wu Y, Henkemeyer M, Yan M. EphB4 forward signalling regulates lymphatic valve development. Nat Commun. 2015;6:6625.

55. Jonason JH, O'Keefe RJ. Isolation and culture of neonatal mouse calvarial osteoblasts. In Skeletal Development and Repair: Methods and Protocols. Hilton MJ, ed. Methods in Molecular Biology 1130. New York, New York, USA: Humana Press. 2014;295-305.

56. Reymond N, d’Água BB, Ridley AJ. Crossing the endothelial barrier during metastasis. Nat Rev Cancer. 2013;13(12):858-870.

57. Salvucci O, et al. EphrinB2 controls vessel pruning through STAT1-JNK3 signalling. Nat Commun. 2015;6:6576.

58. Noberini R, et al. PEGylation potentiates the effectiveness of an antagonistic peptide that targets the EphB4 receptor with nanomolar affinity. PLoS One. 2011;6(12):e28611.

59. Noren NK, Yang NY, Silldorff M, Mutyala R, Pasquale EB. Ephrin-independent regulation of cell substrate adhesion by the EphB4 receptor. Biochem J. 2009;422(3):433-442.

60. Chung K, et al. Structural and molecular interrogation of intact biological systems. Nature. 2013;497(7449):332-337. 